TRANSACTIONS OF THE

AMERICAN MATHEMATICAL SOCIETY

Volume 350, Number 5, May 1998, Pages 1823-1850

S 0002-9947(98)02123-0

\title{
KRONECKER CONJUGACY OF POLYNOMIALS
}

\author{
PETER MÜLLER
}

\begin{abstract}
Let $f, g \in \mathbb{Z}[X]$ be non-constant polynomials with integral coefficients. In $1968 \mathrm{H}$. Davenport raised the question as to when the value sets $f(\mathbb{Z})$ and $g(\mathbb{Z})$ are the same modulo all but finitely many primes. The main progress until now is M. Fried's result that $f$ and $g$ then differ by a linear substitution, provided that $f$ is functionally indecomposable. We extend this result to polynomials $f$ of composition length 2 . Also, we study the analog when $\mathbb{Z}$ is replaced by the integers of a number field. The above number theoretic property translates to an equivalent property of subgroups of a finite group, known as Kronecker conjugacy, a weakening of conjugacy which has been studied by various authors under different assumptions and in other contexts.

We also give a simplified and strengthened version of the Galois theoretic translation to finite groups.
\end{abstract}

\section{INTRODUCTION}

Let $f \in \mathbb{Z}[X]$ be a polynomial with integral coefficients. For a prime $p \in \mathbb{P}$ denote by $\mathcal{V}_{p}(f)$ the value set $f(\mathbb{Z})$ modulo $p$. Suppose there is another polynomial $g \in \mathbb{Z}[X]$ such that

$$
\mathcal{V}_{p}(f)=\mathcal{V}_{p}(g) \text { for all but finitely many } p \in \mathbb{P} .
$$

We call the pair $f, g$ Kronecker conjugate over $\mathbb{Q}$, a notion which will be justified

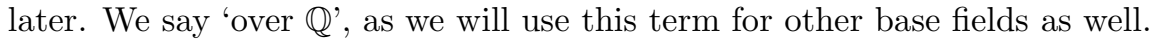

An obvious instance for this to happen is that $g(X)=f(u X+v)$ for some $u \in \mathbb{Q} \backslash\{0\}, v \in \mathbb{Q}$ - then $\mathcal{V}_{p}(f)=\mathcal{V}_{p}(g)$ for all $p$ which do not divide the denominator or numerator of $u$. In such a case, we say that $f$ and $g$ are linearly related over $\mathbb{Q}$. If however $g$ is not linearly related over $\mathbb{Q}$ to $f$, then we say that $f$ and $g$ are properly Kronecker conjugate over $\mathbb{Q}$.

H. Davenport posed the problem of determining properly Kronecker conjugate pairs of polynomials over $\mathbb{Q}$.

This problem can be seen as a far-reaching generalization of another question from algebraic number theory that has already been treated several times. Namely for $n \in \mathbb{N}$, determine those integers $u \in \mathbb{Z}$ which are an $n$-th power modulo all but finitely many primes. If we set $f(X)=u X^{n}, g(X)=X^{n}$, then the hypothesis is equivalent to the one in Davenport's question. In this special case, one can prove that $u$ is an $n$-th power, except for the cases that $n$ is divisible by 8 and

Received by the editors January 16, 1996.

1991 Mathematics Subject Classification. Primary 11C08, 20B10; Secondary 11R09, 12E05, 12F10, 20B20, 20D05.

The author thanks the Deutsche Forschungsgemeinschaft (DFG) for its support in the form of a postdoctoral fellowship. 
$u=w^{n} 2^{n / 2}$ for a non-zero integer $w$ (see [32], [1], [17]). Thus if $h \in \mathbb{Z}[X]$ is an arbitrary polynomial of positive degree, then $f(X)=h\left(X^{8}\right)$ and $g(X)=h\left(16 X^{8}\right)$ are properly Kronecker conjugate over $\mathbb{Q}$. To date, no other examples are known (up to trivial modifications).

A non-existence result has been given by M. Fried. To state it we need to introduce some more terminology. Let $K$ be a field of characteristic 0 . We call a polynomial $h \in K[X]$ of degree $\geq 2$ indecomposable, if it cannot be written as a composition of polynomials of lower degree over $K$. For any polynomial $f \in K[X]$ of degree $\geq 2$ write $f(X)=f_{1}\left(f_{2}\left(\ldots f_{l}(X) \ldots\right)\right)$ with indecomposable polynomials $f_{i} \in K[X]$. By a classical result of Ritt (see [27], [21]), the number $l$ is independent of the decomposition. We say that $f$ has composition length $l$. It is known (see $[13,3.5])$ that the polynomials $f_{i}$ are indecomposable over any field extension of $K$. Thus the composition length of a polynomial is independent of the base field.

Now we can state Fried's result. Putting [9, Section 2] and [8, Section 3] together, one obtains

Theorem 1.1 (Fried). There is no pair $f, g \in \mathbb{Z}[X]$ of properly Kronecker conjugate polynomials over $\mathbb{Q}$ with $f$ being indecomposable.

One of the aims in this paper is to obtain the same result for composition length 2 .

Theorem 1.2. There is no pair $f, g \in \mathbb{Z}[X]$ of properly Kronecker conjugate polynomials over $\mathbb{Q}$ with $f$ having composition length 2 .

As remarked already, $f(X)=X^{8}, g(X)=16 X^{8}$ is a pair of properly Kronecker conjugate polynomials with $f$ having composition length 3 . So Theorem 1.2 does not extend to higher composition lengths without change.

There is an obvious generalization of Davenport's original question. Let $K$ be a number field, and denote by $\mathcal{O}_{K}$ its ring of integers. Now, replace the condition of Kronecker conjugacy for $f, g \in \mathcal{O}_{K}[X]$ by

$$
\mathcal{V}_{\mathfrak{p}}(f)=\mathcal{V}_{\mathfrak{p}}(g) \text { for all but finitely many non-zero prime ideals } \mathfrak{p} \text { of } \mathcal{O}_{K} \text {. }
$$

The picture changes if we allow $K$ to be bigger than $\mathbb{Q}$. New examples occur, which even share a strong property. Let $f, g \in K[X]$ be properly Kronecker conjugate over $K$, and suppose that $f$ and $g$ are even not linearly related over the algebraic closure $\bar{K}$ of $K$. Then we say that $f$ and $g$ are strongly Kronecker conjugate over $K$. For instance, our previous example $f(X)=h\left(X^{8}\right), g(X)=h\left(16 X^{8}\right)$ is not strongly Kronecker conjugate over $\mathbb{Q}$, as $f$ and $g$ are linearly related over $\mathbb{Q}(\sqrt{2})$.

The number field version of Theorem 1.1, obtained by M. Fried [10] together with Feit [5], is

Theorem 1.3 (Fried, Feit). Let $K$ be a number field, and $f, g \in \mathcal{O}_{K}[X]$ be properly Kronecker conjugate over $K$ with $f$ being indecomposable. Then $f$ and $g$ are even strongly Kronecker conjugate, and the degree of $f$ is 7, 11, 13, 15, 21, or 31. For each of these degrees and suitably big $K$, there indeed exist examples.

Remark. We will see in section 2.3 that the degrees of $f$ and $g$ are the same if $f$ and $g$ are Kronecker conjugate.

Again, we extend this theorem to

Theorem 1.4. Let $K$ be a number field, and $f, g \in \mathcal{O}_{K}[X]$ be properly Kronecker conjugate over $K$. Suppose that $f(X)=a(b(X))$ with indecomposable $a, b \in K[X]$, 
such that neither $a$ nor $b$ is strongly Kronecker conjugate over $K$ to another polynomial. Then $f$ and $g$ are strongly Kronecker conjugate, and

$$
(\operatorname{deg} a, \operatorname{deg} b) \in\{(4,2),(6,2),(8,2),(10,2),(5,3),(8,3)\} .
$$

For each of these cases there exist examples $f$ and $g$ over a suitably big $K$; they are explicitly given in section 4 .

So far, we have seen only finitely many possible degrees of properly Kronecker conjugate polynomials. Of course, there is a trivial source for producing more such pairs. Let $d$ and $d^{\prime}$ be properly Kronecker conjugate polynomials, and $h$ be an arbitrary non-constant polynomial. Then also $f(X)=h(d(X)), g(X)=h\left(d^{\prime}(X)\right)$ are Kronecker conjugate, and in general even properly. Adopting a term used by Fried in a similar context, we say that a Kronecker conjugate pair $f, g$ is newly Kronecker conjugate if it does not have this form. In view of the results, one might wonder if there are strongly and newly Kronecker conjugate polynomials of arbitrary high degree. In [23] we give the first infinite series.

Theorem 1.5. Let $m \geq 3$ be an integer, and $\zeta$ be a primitive $4 m$-th root of unity. Set

$$
\begin{aligned}
& f(X)=\left(\left(X^{2}+\left(\zeta^{4}-1\right)\right)^{2}+\zeta^{4}-1\right)^{m}, \\
& g(X)=\left(\left(X^{2}+\zeta^{m-2}\left(\zeta^{4}-1\right)\right)^{2}+\zeta^{4}-1\right)^{m} .
\end{aligned}
$$

Then $f$ and $g$ are strongly and newly Kronecker conjugate over any number field $K$ which contains $\zeta$.

There is another non-existence result [24] which would require some more definitions to state. It allows arbitrary composition lengths, but imposes restrictions on the polynomials $f_{i}$ in a maximal decomposition $f(X)=f_{1}\left(f_{2}\left(\ldots f_{l}(X) \ldots\right)\right)$ of $f$.

None of these results seems to be deducible from the very definition of Kronecker conjugacy. Rather, they depend on an equivalent group theoretic translation of Kronecker conjugacy. Let $f, g \in \mathcal{O}_{K}[X]$. Fix a transcendental $t$ over $K$. Fix a Galois extension $\Pi$ of $K(t)$ which contains elements $x$ and $y$ such that $f(x)-t=0$ and $g(y)-t=0$. Denote by $G$ the Galois group of $\Pi \mid K(t)$, and let $U$ and $V$ be the stabilizers of $x$ and $y$ in $G$ respectively. Fried's basic theorem (see $[12,19.27]$ ) is

Theorem 1.6 (Fried). Let $K$ be a number field, and $f, g \in \mathcal{O}_{K}[X]$ be non-constant polynomials. Then the following are equivalent.

(i) $f$ and $g$ are Kronecker conjugate over $K$.

(ii) $\bigcup_{g \in G} U^{g}=\bigcup_{g \in G} V^{g}$.

Note that condition (ii) is easily seen to be independent of the chosen Galois extension $\Pi$ of $K(t)$. In section 2.1 we will give a simple proof of Theorem 1.6. Actually, our approach allows us to give more information, namely to determine the prime ideals where $\mathcal{V}_{\mathfrak{p}}(f)=\mathcal{V}_{\mathfrak{p}}(g)$ might fail to hold.

The group theoretic condition (ii) first appeared in the work of Kronecker on characterizing finite extension of number fields by the splitting behavior of prime ideals. See [19], [17], [12, Section 19.5]. This condition has also been investigated (independently of its number theoretical context) by various group theorists. See [15], [26], [29]. We call two subgroups $U$ and $V$ of an abstract finite group $G$ Kronecker conjugate if (ii) holds. 
We want to give one example to show that Kronecker conjugacy in finite groups gives rise to tough questions. Suppose that (ii) holds and $U$ has index 2 in $G$. Then clearly $V \leq U \triangleleft G$, and the question is whether $U=\bigcup_{g \in G} V^{g}$ forces $U=V$. (This is a generalization of the easy fact that a finite group cannot be the union of the conjugates of a proper subgroup.) Yet, this question is distinctly non-trivial and has been answered in the affirmative by Saxl [29] (and generalized by Guralnick [15]). Note that if we assume $U \triangleleft G$ of index 3, then (ii) does not force $U=V$ any more. An example is the alternating groups $G=\mathrm{A}_{4}, U$ the Sylow 2-subgroup of $G$, and $V$ a subgroup of order 2 in $U$.

In our context of polynomials, we gain additional group theoretic information, which makes some questions more tractable. The next section is devoted to providing all this information needed to prove Theorems 1.2 and 1.4 in section 3 . We compute the polynomials whose existence is claimed in Theorem 1.4 in section 4 . We finish by stating two conjectures in section 5 .

Finally, we note that there is another classical question which has the same arithmetic flavor as Davenport's problem. In 1923 I. Schur [31] investigated the question of when the value set of $f \in \mathbb{Z}[X]$ is the full residue system modulo $p$ for infinitely many primes $p$. This problem has been solved by M. Fried in [7]. For more literature and related results, see the recent exposition in [33].

\section{KRONECKER CONJUGACY AND MONODROMY GROUPS}

This section is devoted to the passage from the original question to an equivalent question about finite groups, first giving consequences from Kronecker conjugacy, and providing results about monodromy groups of polynomials to be used later. We keep the notation from the Introduction.

2.1. Translation to finite groups. We give a short proof of Theorem 1.6 different from the original proof of Fried (which uses Cebotarev's density theorem for function fields over finite fields; see [9, Section 2]) and the model theoretic proof in [12], which gives a far more general principle for characteristic transfer. Actually, our argument allows for a stronger statement, namely the implication (i) $\Rightarrow$ (iii) in Theorem 2.3 below.

The argument is based on

Theorem 2.1 (Frobenius). Let $h \in \mathcal{O}_{K}[X]$ be monic and separable, and assume that $h(X) \equiv 0(\bmod \mathfrak{p})$ has a solution in $\mathcal{O}_{K}$ for almost all non-zero prime ideals $\mathfrak{p}$ of $\mathcal{O}_{K}$. Then every element of the Galois group of $h(X)$ over $K$ fixes at least one root of $h$.

A proof of this theorem, which follows from Frobenius' density theorem (a weaker form of Čebotarev's density theorem) can be found in [12].

We need a converse of Frobenius' theorem. As we could not find it in the literature, we supply a proof.

Proposition 2.2. Let $h \in \mathcal{O}_{K}[X]$ be monic and separable, and assume that every element of the Galois group of $h(X)$ over $K$ fixes at least one root of $h$. Then $h(X) \equiv 0(\bmod \mathfrak{p})$ has a solution in $\mathcal{O}_{K}$ for every non-zero prime ideal $\mathfrak{p}$ of $\mathcal{O}_{K}$.

Proof. Let $L$ be a splitting field of $h(X)$ over $K$. Let $\mathcal{O}_{L}$ be the integral closure of $\mathcal{O}_{K}$ in $L$, and $\mathfrak{P}$ be a prime ideal of $\mathcal{O}_{L}$ lying over $\mathfrak{p}$. The roots of $h(X)=0$ lie in $\mathcal{O}_{L}$ by the assumption about $h$. Let $D$ and $I$ be the decomposition and inertia 
group of $\mathfrak{P}$ respectively. Then $D / I$ is cyclic, and maps isomorphically to the Galois group of the extension $\mathcal{O}_{L} / \mathfrak{P} \mid \mathcal{O}_{K} / \mathfrak{p}$ of residue fields. Pick $d \in D$ such that the coset $d I$ generates $D / I$. By the assumption, $d$ fixes a root $\alpha$ of $h(X)=0$. Thus $d I$ fixes the image of $\alpha$ in $\mathcal{O}_{L} / \mathfrak{P}$. But $d I$ generates the full Galois group of $\mathcal{O}_{L} / \mathfrak{P} \mid \mathcal{O}_{K} / \mathfrak{p}$, so there is $\beta \in \mathcal{O}_{K}$ which is congruent to $\alpha$ modulo $\mathfrak{P}$. This gives $h(\beta) \in \mathfrak{P} \cap \mathcal{O}_{K}=\mathfrak{p}$, and the assertion follows.

A refinement of Theorem 1.6 is

Theorem 2.3. Let $K$ be a number field, and $f, g \in \mathcal{O}_{K}[X]$ be non-constant polynomials. Let $d$ be the product of the leading coefficients of $f$ and $g$. Then the following are equivalent.

(i) $\mathcal{V}_{\mathfrak{p}}(f)=\mathcal{V}_{\mathfrak{p}}(g)$ for all but finitely many non-zero prime ideals $\mathfrak{p}$ of $\mathcal{O}_{K}$; that is, $f$ and $g$ are Kronecker conjugate over $K$.

(ii) $\bigcup_{g \in G} U^{g}=\bigcup_{g \in G} V^{g}$.

(iii) $\mathcal{V}_{\mathfrak{p}}(f)=\mathcal{V}_{\mathfrak{p}}(g)$ for all non-zero prime ideals $\mathfrak{p}$ of $\mathcal{O}_{K}$ which do not divide $d$.

Proof. Suppose that (i) holds. We show (ii). Let $n$ be the degree of $f$, and $u$ be the coefficient of $X^{n}$ in $f$. Note that Kronecker conjugacy is preserved when we replace $f(X)$ and $g(X)$ by $u^{n-1} f(X / u)$ and $u^{n-1} g(X)$ respectively. Thus we may assume that $f$ is monic. Now, for $a \in \mathcal{O}_{K}$, the hypothesis says that $f(X)-g(a) \equiv 0$ $(\bmod \mathfrak{p})$ has a root for almost all non-zero prime ideals $\mathfrak{p}$ of $\mathcal{O}_{K}$. It is clear that there are only finitely many $a \in \mathcal{O}_{K}$ such that $f(X)-g(a)$ is not separable, as those $a$ are the roots of the discriminant of $f(X)-g(Y)$ taken with respect to $X$, which is a polynomial of positive degree in $Y$.

Hilbert's irreducibility theorem (see [34] or [12]) tells us that the Galois groups $\operatorname{Gal}(f(X)-g(y) \mid K(y))$ and $\operatorname{Gal}(f(X)-g(a) \mid K)$ are isomorphic as permutation groups on the roots of $f(X)-g(y)$ and $f(X)-g(a)$ respectively for infinitely many $a \in \mathcal{O}_{K}$. Recall that $g(y)=t$. Thus every element of the Galois group $\operatorname{Gal}(f(X)-t \mid K(y))$ fixes at least one root. This Galois group is just the induced action of $V$ on the roots of $f(X)-t$. ( $V$ need not act faithfully on these roots.) Hence every element in $V$ fixes a root. But these roots are the conjugates of $x$, and the stabilizer of $x$ is $U$. So every element of $V$ lies in some conjugate of $U$. Now (ii) follows from symmetry.

Of course, (iii) implies (i), so we are left to show the implication (ii) $\Rightarrow$ (iii). Again, let $u$ be the coefficient of $X^{n}$ of the degree $n$ polynomial $f$. We are going to show that $\mathcal{V}_{\mathfrak{p}}(f) \supseteq \mathcal{V}_{\mathfrak{p}}(g)$ for all non-zero prime ideals $\mathfrak{p}$ of $\mathcal{O}_{K}$ which do not divide $u$. The assertion then follows from symmetry. So fix a non-zero prime ideal $\mathfrak{p}$ of $\mathcal{O}_{K}$ which does not divide $u$. Again, replace $f(X)$ by $u^{n-1} f(X / u)$ and $g(X)$ by $u^{n-1} g(X)$. This does not affect (ii) and (iii). Let $a \in \mathcal{O}_{K}$ be arbitrary. We want to show that $f(X)-g(a) \equiv 0(\bmod \mathfrak{p})$ has a solution. By adding some element from $\mathfrak{p}$ to $a$, we assume that $f(X)-g(a)$ is separable. So the Galois group of $f(X)-g(a)$ over $K$ is a subgroup of the Galois group of $f(X)-t=f(X)-g(y)$ over $K(y)$. Every element of the latter Galois group fixes a root of $f(X)-t$, so this is even more true for the former Galois group acting on the roots of $f(X)-g(a)$. Now Proposition 2.2 yields the assertion.

For future use we draw the following conclusion. 
Corollary 2.4. Let $K$ be a number field, and $f, g \in \mathcal{O}_{K}[X]$ be non-constant and monic polynomials which are Kronecker conjugate over $K$. Then $\mathcal{V}_{\mathfrak{p}}(f)=\mathcal{V}_{\mathfrak{p}}(g)$ for all non-zero prime ideals $\mathfrak{p}$ of $\mathcal{O}_{K}$.

2.2. Monodromy groups of polynomials. Here we record some properties of monodromy groups of polynomials which are independent of the setup of Kronecker conjugacy. For a similar exposition see also [11]. Let $K$ be a field of characteristic 0 , and $f \in K[X]$ a polynomial of degree $n \geq 1$. Throughout this section, we can assume without loss of generality that $f$ is monic. We denote by $\bar{K}$ an algebraic closure of $K$, and let $t$ be a transcendental over $K$. We say that the Galois group $G$ of $f(X)-t$ over $K(t)$ is the arithmetic monodromy group of $f$, and call the Galois group $\dot{G}$ of $f(X)-t$ over $\bar{K}(t)$ the geometric monodromy group of $f$. Both groups are regarded as permutation groups on the roots of $f(X)-t$, and under this identification as permutation groups, $\dot{G}$ is a normal subgroup of $G$. The factor group $G / \dot{G}$ has a natural interpretation. Let $\Pi$ be a splitting field of $f(X)-t$ over $K(t)$; thus $G=\operatorname{Gal}(\Pi \mid K(t))$. Let $\hat{K}$ be the algebraic closure of $K$ in $\Pi$. As $\bar{K}(t) \cap \Pi=\hat{K}(t)$ (see [3, Corollary 2, V, $\S 4]$ ), we have $\dot{G}=\operatorname{Gal}(\Pi \mid \hat{K}(t))$; thus $G / \dot{G}$ can be identified with the Galois group $\operatorname{Gal}(\hat{K} \mid K)$.

We are now going to define two important subgroups of $G$, which also give us a hold on the extension $\hat{K} \mid K$. For this we make the change of variables $Y=1 / X$ and $z=1 / t$. Then the equation $f(X)-t=0$ can be written as

$$
Y^{n}-z \tilde{f}(Y)=0,
$$

where $\tilde{f}$ is the reciprocal polynomial of $f$. Note that $\tilde{f}$ has constant term 1 . The Eisenstein criterion shows that the left hand side is irreducible even over the power series field $\bar{K}((z))$. In particular, $G$ and its normal subgroup $\dot{G}$ are transitive. A splitting field of $Y^{n}-z \tilde{f}(Y)$ over $K((z))$ has the form $\Pi K((z))$. We can describe this splitting field explicitly. For this let $z^{1 / n}$ be an $n$-th root of $z$, and set $W=Y / z^{1 / n}$. Then the above equation becomes

$$
W^{n}-\tilde{f}\left(W z^{1 / n}\right)=0 .
$$

Hensel's lemma tells us that this equation has a solution in $K\left[\left[z^{1 / n}\right]\right]$. Thus, there is

$$
y_{0}=z^{1 / n}+a_{2} z^{2 / n}+a_{3} z^{3 / n}+\ldots \in K\left[\left[z^{1 / n}\right]\right]
$$

with

$$
y_{0}^{n}-z \tilde{f}\left(y_{0}\right)=0 .
$$

The other solutions $y_{i}$ are given by replacing $z^{1 / n}$ by $\zeta^{i} z^{1 / n}$, where $\zeta$ is a fixed primitive $n$-th root of unity. We get that $K(\zeta)\left(\left(z^{1 / n}\right)\right)$ is a splitting field of $Y^{n}-$ $z \tilde{f}(Y)$ over $K((z))$. Hence the field $\hat{K}$ is a subfield of $K(\zeta)$. Set

$$
D=\operatorname{Gal}\left(K(\zeta)\left(\left(z^{1 / n}\right)\right) \mid K((z))\right),
$$

and

$$
Z=\operatorname{Gal}\left(K(\zeta)\left(\left(z^{1 / n}\right)\right) \mid K(\zeta)((z))\right) .
$$

The group $Z$ is cyclic of order $n$, and permutes the elements $y_{i}$ cyclically. Also, the structure of $D$ is clear. It is the semidirect product of $Z$ with $\operatorname{Gal}(K(\zeta) \mid K)$, where 
the action is given by identifying $Z$ with $\mathbb{Z} / n \mathbb{Z}$ and $\operatorname{Gal}(K(\zeta) \mid K)$ with a subgroup of the group of units of $\mathbb{Z} / n \mathbb{Z}$ in its action by multiplication on $\mathbb{Z} / n \mathbb{Z}$.

It is clear that $D$ and $Z$ are naturally identified as subgroups of $G$. As the group $D$ induces $\operatorname{Gal}(K(\zeta) \mid K)$ on $K(\zeta)$, it also induces $\operatorname{Gal}(\hat{K} \mid K)$ on $\hat{K}$. Hence $G=\dot{G} D$. Further, $Z \leq \dot{G}$, as $Z$ is trivial on $\hat{K} \leq K(\zeta)$. Therefore $D / Z$ maps surjectively on $G / \dot{G}$. A particular case of interest is $K=\mathbb{Q}$. Then $D / Z$ is the full group of units of $\mathbb{Z} / n \mathbb{Z}$.

We summarize:

Proposition 2.5. With the above notation, let $G$ and $\dot{G}$ act on the $n$ roots of $f(X)-t$. Then $\dot{G}$ contains a regular cyclic subgroup $Z$ of order $n$. Further, there is a subgroup $D$ of $G$ with $Z \leq D$, such that $Z$ has a complement in $D$ which is isomorphic to a subgroup of the group $Z_{n}^{\star}$ of units of $\mathbb{Z} / n \mathbb{Z}$. If $K=\mathbb{Q}$, then this complement is isomorphic to $Z_{n}^{\star}$. Furthermore, $|G / \dot{G}|$ divides $\left|Z_{n}^{\star}\right|$.

The presence of the cyclic regular subgroup $Z$ of $G$ has an interesting consequence, which we state in a slightly more general form. For a subgroup $M$ of a finite group $H$, denote by $\operatorname{core}_{H}(M)$ the group $\bigcap_{h \in H} M^{h}$. Thus core ${ }_{H}(M)$ is the biggest subgroup of $M$ which is normal in $H$.

Lemma 2.6. Let $U$ be a subgroup of a finite group $H$, such that $H=Z U$ with an abelian subgroup $Z$ of $H$. If $U<M \leq H$, then $M \cap Z \leq \operatorname{core}_{G}(M)$, and $M \cap Z$ is not contained in $U$.

Proof. As $H=Z U$, we have $M=U(M \cap Z)$, so $M \cap Z$ is not contained in $U$. Further, the set of $H$-conjugates of $M$ coincides with the set of $Z$-conjugates of $M$. Hence $M \cap Z=\bigcap_{z \in Z}(M \cap Z)^{z} \leq \bigcup_{z \in Z} M^{z}=\operatorname{core}_{H}(M)$.

Let $U$ be the stabilizer in $G$ of the root $x$ of $f(X)-t$. There is a good correspondence between the maximal chains of subgroups from $U$ to $G$ and decompositions of $f$ into indecomposable polynomials over $K$. The proof of the following lemma follows readily from Lüroth's theorem (see also [13]).

Lemma 2.7. Let $f(X)=f_{1}\left(f_{2}\left(\ldots\left(f_{l}(X)\right) \ldots\right)\right)$ be a decomposition of $f$ into indecomposable polynomials $f_{i}$. Denote by $U_{i}$ the stabilizer of $f_{i}\left(f_{i+1}\left(\ldots\left(f_{l}(x)\right) \ldots\right)\right)$ in $G$ for $1 \leq i \leq l$. Then $G=U_{1}>U_{2}>\ldots>U_{l}>U$ is a maximal chain of subgroups.

Conversely, if such a chain of subgroups is given, then there is a corresponding decomposition of $f$.

In particular, $G$ is a primitive permutation group if and only if $f$ is indecomposable.

Therefore the knowledge of the monodromy groups of indecomposable polynomials is of basic importance. In order to formulate a classification result, we need to introduce some terminology. 

$\mathrm{A}_{n}, \mathrm{~S}_{n}$
Alternating or symmetric group of degree $n$;
$C_{n}$
Cyclic group of degree $n$;
$D_{n}$
Dihedral group of degree $n$ (and size $2 n$ if $n>2$, and size 2 if $n=2)$;
$M_{n} \quad$ Mathieu group of degree $n$;
$\mathrm{PGL}_{m}(q)$
Projective linear group over the field with $q$ elements, acting on the points of the $(m-1)$-dimensional projective space;
$\operatorname{PSL}_{m}(q) \quad$ Projective special linear subgroup of $\operatorname{PGL}_{m}(q)$;
$\mathrm{P}_{m}(q) \quad$ Projective semi-linear group. This group is generated by $\mathrm{PGL}_{m}(q)$ and the action of $\operatorname{Aut}\left(\mathbb{F}_{q}\right)$ on the projective space, where $\mathbb{F}_{q}$ is the finite field with $q$ elements;
$\operatorname{AGL}_{1}(n) \quad$ Affine group $C_{n} \rtimes \operatorname{Aut}\left(C_{n}\right)$. We regard it as the group of mappings on $\mathbb{Z} / n \mathbb{Z}$ given by $r \mapsto a r+b$ for $b \in \mathbb{Z} / n \mathbb{Z}$ and $a$ a unit of $\mathbb{Z} / n \mathbb{Z}$.

The following result is basic for the rest of the paper.

Proposition 2.8. Let $K$ be a number field, and $f \in K[X]$ an indecomposable polynomial of degree $n$ which is not strongly Kronecker conjugate to another polynomial. Then, with the previous notation, one of the following holds.

(1) $n \in \mathbb{P}, \dot{G}=C_{n}, C_{n} \leq G \leq \mathrm{AGL}_{1}(n)$;

(2) $n \in \mathbb{P}, \dot{G}=D_{n}, D_{n} \leq G \leq \operatorname{AGL}_{1}(n)$;

(3) $n$ odd, $\dot{G}=\mathrm{A}_{n}, \mathrm{~A}_{n} \leq G \leq \mathrm{S}_{n}$;

(4) $\dot{G}=G=\mathrm{S}_{n}$;

(5) $n=6, \dot{G}=G=\mathrm{PGL}_{2}(5)$;

(6) $n=8, \dot{G}=G=\mathrm{PGL}_{2}(7)$;

(7) $n=9, \dot{G}=G=\mathrm{P \Gamma L}_{2}(8)$;

(8) $n=10, \dot{G}=G=\mathrm{P \Gamma L}_{2}(9)$;

(9) $n=11, \dot{G}=G=\mathrm{M}_{11}$, or $n=23, \dot{G}=G=\mathrm{M}_{23}$.

Proof. The group $\dot{G}$ equals the Galois group of $f(X)-t$ over $\mathbb{C}(t)$. These Galois groups have been classified when $f$ is indecomposable by Feit ([5]; see [21] for a correction) for the case that $f$ has a strongly Kronecker conjugate partner, and in [21] for the other cases. So [21] gives us the possibilities for $\dot{G}$. The statement about $G$ follows from $\dot{G} \unlhd G$ and considering the normalizer of $\dot{G}$ in $\mathrm{S}_{n}$, where $\dot{G}$ has degree $n$.

Remark. Though we won't need the result, we want to give the complementary list of monodromy groups of indecomposable polynomials. Suppose that $f$ is indecomposable and has a strongly Kronecker conjugate partner. Then $\dot{G}=G$, and $G$ is one of the following groups of degree $n$ : $\mathrm{PSL}_{2}(11)(n=11), \mathrm{PGL}_{3}(2)(n=7)$, $\mathrm{PGL}_{3}(3)(n=13), \mathrm{PGL}_{4}(2)(n=15), \mathrm{P}_{3}(4)(n=21)$, and PGL $(2)(n=31)$.

2.2.1. The geometric interpretation of $\dot{G}$. As a preparation for the explicit computations in section 4 and some arguments needed in section 3.3, we say something about the geometric interpretation of $\dot{G}$ as a monodromy group of a branched cover. This, by the way, is also the tool to prove the above proposition. 
Let $f$ have degree $n$. Then $f$ defines a branched covering of Riemann spheres $\mathbb{P}^{1} \rightarrow \mathbb{P}^{1}$ by sending $z$ to $f(z)$. We call this cover $f$ also.

Let $\mathcal{B}=\left\{b_{1}, b_{2}, \ldots, b_{r}\right\}$ be the set of branch points of $f$. These are the points $b$ with $\left|f^{-1}(b)\right|<n$. Without loss, let $b_{r}$ be the branch point $\infty$. The branch points different from $\infty$ will be called finite branch points. Fix $p \in \mathbb{P}^{1} \backslash \mathcal{B}$, and denote by $\pi_{1}$ the fundamental group $\pi_{1}\left(\mathbb{P}^{1} \backslash \mathcal{B}, p\right)$. Then $\pi_{1}$ acts transitively on the points of the fiber $f^{-1}(p)$ by lifting of paths. We fix a numbering $1,2, \ldots, n$ of this fiber. So we get a homomorphism $\pi_{1} \rightarrow \mathrm{S}_{n}$. By standard arguments (see [22, Section 2] for a self-contained presentation), the image of $\pi_{1}$ can be identified with the geometric monodromy group $\dot{G}$; thus we write $\dot{G}$ for this group too.

We choose a standard homotopy basis for $\pi_{1}=\pi_{1}\left(\mathbb{P}^{1} \backslash \mathcal{B}, p\right)$ as follows. Let $\gamma_{i}$ be represented by paths which start and end in $p$ and wind once around $b_{i}$ counterclockwise, and around no other branch point, such that $\gamma_{1} \gamma_{2} \cdots \gamma_{r}=1$. Then $\gamma_{1}, \gamma_{2}, \ldots, \gamma_{r-1}$ freely generate $\pi_{1}$.

Definition. For $\sigma \in \mathrm{S}_{n}$, let $e_{1}, \ldots, e_{m}$ be the cycle lengths of $\sigma$. Define the index of $\sigma$ by ind $\sigma=\sum_{j=1}^{m}\left(e_{j}-1\right)$.

Let $\sigma_{i}$ be the image of $\gamma_{i}$ in $\mathrm{S}_{n}$. If the points $s_{1}, \ldots, s_{m}$ in the fiber of $b_{i}$ have multiplicities $e_{1}, \ldots, e_{m}$, respectively, then $\sigma_{i}$ has cycle lengths $e_{1}, \ldots, e_{m}$. In particular, ind $\sigma_{i}=n-\left|\pi^{-1}\left(b_{i}\right)\right|$. Further, $\sigma_{r}$ is an $n$-cycle, because $b_{r}=\infty$ has only one preimage under $f$. As $\mathbb{P}^{1}$ has genus 0 , and $\sigma_{r}$ has index $n-1$, the Riemann-Hurwitz genus formula gives (setting $R=r-1$ ).

$\dot{G}$ is generated by $\sigma_{1}, \ldots, \sigma_{R}$, and the following holds:

$$
\left\{\begin{array}{l}
\sigma_{1} \sigma_{2} \cdots \sigma_{R} \text { is an } n \text {-cycle; } \\
\sum_{i=1}^{R} \operatorname{ind} \sigma_{i}=n-1 .
\end{array}\right.
$$

The tuple $\left(\sigma_{1}, \sigma_{2}, \ldots, \sigma_{R}\right)$ will be called a genus 0 system of the cover $f$, or of the group $\dot{G}$. Conversely, the hypothesis about a finite permutation group of degree $n$ having a generating system as above will be called the genus 0 condition.

We may reorder the conjugacy classes in $\dot{G}$ that the $\sigma_{i}$ belong to. Namely, if we replace the $i$-th and $(i+1)$-st position in $\left(\sigma_{1}, \sigma_{2}, \ldots, \sigma_{r}\right)$ by $\sigma_{i+1}$ and $\sigma_{i}^{\sigma_{i+1}}$ respectively, then the above properties are preserved. (Geometrically, that amounts of giving the branch points a different order before choosing the standard generators of $\pi_{1}$.)

From Riemann's existence theorem, we get a converse to this procedure. Let a finite permutation group of degree $n$ be generated by $\sigma_{1}, \sigma_{2}, \ldots, \sigma_{R}$ such that ( $\star$ ) holds. Then there is a polynomial over some number field, such that $\dot{G}$ is just the group we started with, and the genus 0 system is given by $\left(\sigma_{1}, \sigma_{2}, \ldots, \sigma_{R}\right)$.

We say that a polynomial $f$ equals $\tilde{f}$ up to linear changes if there are linear polynomials $L_{1}, L_{2}$ over $\mathbb{C}$ such that $f(X)=L_{1}\left(\tilde{f}\left(L_{2}(X)\right)\right)$. The Chebyshev polynomial $T_{n}(X) \in \mathbb{Q}[X]$ of degree $n$ is defined by the relation $T_{n}(Z+1 / Z)=Z^{n}+1 / Z^{n}$. Note that $T_{m n}(X)=T_{m}\left(T_{n}(X)\right)$, hence the indecomposable Chebyshev polynomials have prime degree. The following lemma follows mostly from [21] and some easy computations.

Lemma 2.9. Let $f \in K[X]$ be indecomposable of degree $n$.

(i) If $\dot{G}=C_{n}$, then $f$ equals $X^{n}$ up to linear changes, and $n$ is a prime. 
(ii) If $\dot{G}=D_{n}$, then $f$ equals $T_{n}$ up to linear changes, and $n$ is a prime. If $n>2$, then the branch points of $T_{n}$ are -2 and 2 . The elements $\sigma_{1}$ and $\sigma_{2}$ associated to these branch points are both involutions with exactly one fixed point.

(iii) If $\dot{G}=\mathrm{S}_{4}$, then either there are three finite branch points, and the associated $\sigma_{i}$ are transpositions, or there are two finite branch points, and the associated $\sigma_{i}$ are one transposition and one 3 -cycle.

2.3. Group theoretic consequences from Kronecker conjugacy. The notion of Kronecker conjugacy as in Theorem 1.6(ii) makes sense over any field $K$ of characteristic 0 . Thus assume that $f, g$ are non-constant polynomials in $K[X]$. Again, use the setup preceding Theorem 1.6. We begin with an easy lemma.

Lemma 2.10. The polynomials $f$ and $g$ are linearly related over $K$ if and only if $U$ and $V$ are conjugate in $G$.

Proof. Suppose that $f(X)=g(u X+v)$. Then $g(u x+v)=t=g(y)$; thus $u x+v$ and $y$ are conjugate elements, so their respective stabilizers $U$ and $V$ are conjugate. For the converse, assume $U=V^{h}$ for some $h \in G$. Then $t=g(y)=g\left(y^{h}\right)$ and $y^{h}$ is fixed by $U$, so it lies in $K(x)$. Thus $f(x)=g(R(x))$ for some rational function $R \in K(X)$. On the other hand, the degrees of $f$ and $g$ are the same (both equal the index $[G: U]$ ), so $R$ has degree 1 . Looking at the denominator shows that $R$ is a polynomial, and the claim follows.

Lemma 2.11. $G$ contains a cyclic subgroup $Z$ such that $G=U Z=V Z$.

Proof. This follows either from a slight extension of the argument yielding Proposition 2.5, or from the geometric description of geometric monodromy groups as in section 2.2. Or see the proof of $[12,19.29]$.

Lemma 2.12. If $f, g$ are Kronecker conjugate over $K$, then $f$ and $g$ are Kronecker conjugate over any finite extension of $K$.

Proof. Galois theoretically, this translates to the condition that for a subgroup $H$ of $G$ which still acts transitively on the coset spaces $G / U$ and $G / V$, the subgroups $U \cap H$ and $V \cap H$ are Kronecker conjugate in $H$, which is obvious.

Lemma 2.13. Let $A$ and $B$ be Kronecker conjugate subgroups of a finite group $H$. Suppose there is an abelian subgroup $Z$ of $H$ with $H=A Z=B Z$. Then $A \cap Z=B \cap Z$. Thus $|A|=|B|$. Furthermore, $\operatorname{core}_{H}(A)=\operatorname{core}_{H}(B)$.

Proof. By the argument from the previous proof, $A \cap Z$ and $B \cap Z$ are Kronecker conjugate in $Z$; hence they are equal. Let $N$ be the core of $A$ in $H$. Then $A=A N$ and $B N$ are Kronecker conjugate in $H$. Thus $A \cap Z=B \cap Z=B N \cap Z$ by the first part of the lemma. The modular law yields $B N=B N \cap B Z=B(B N \cap Z)=$ $B(B \cap Z)=B$, so $N \leq B$. From symmetry we get the assertion.

For the following development, we assume that the Galois extension of $K(t)$ which contains $x$ and $y$ is taken to be minimal. The preceding lemma shows that if $f$ and $g$ are Kronecker conjugate over $K$, then $U$ and $V$ have trivial core in $G$; that is, $G$ acts faithfully on the conjugates of $x$ as well as on the conjugates of $y$. This also has an implication for the geometric consideration in section 2.2.

Lemma 2.14. If $f$ and $g$ are Kronecker conjugate over $K$, then the branch points of $f$ and $g$ coincide. 
Proof. This follows from the preceding remark. Namely let $\mathcal{B}_{f}$ and $\mathcal{B}_{g}$ be the branch points of $f$ and $g$ respectively. For $p \in \mathbb{P}^{1} \backslash\left(\mathcal{B}_{f} \cup \mathcal{B}_{g}\right)$ choose generators $\sigma_{i}$ of $\pi_{1}\left(\mathbb{P}^{1} \backslash\left(\mathcal{B}_{f} \cup \mathcal{B}_{g}\right), p\right)$ similarly as in section 2.2. If $b$ is a branch point of $f$, then the associated $\sigma_{i}$ is not trivial on the coset space $G / U$, so it is also not trivial on the coset space $G / V$. That means $b$ is a branch point of $g$ as well. From symmetry we get the claim.

2.4. Cases of Kronecker conjugacy. Here we present the general type of the new cases of Kronecker conjugacy which belong to Theorem 1.4. In section 3 we will be able to identify $G$ and a subgroup $M$ such that subgroups $U$ and $V$ of $M$ are Kronecker conjugate in $G$. While we will exactly determine $G$ and $M$, we still have to determine what $U$ and $V$ really look like. The technical Proposition 2.16 will take care of that.

Let $\mathbb{F}_{q}$ be a finite field with $q$ elements, and let $\Gamma L_{2}(q)$ be the semilinear group over $\mathbb{F}_{q}$. Write $\Gamma \mathrm{L}=\operatorname{Aut}\left(\mathbb{F}_{q}\right) \ltimes \mathrm{GL}_{2}(q)$, and view this group as acting from the right on $\mathbb{F}_{q}^{2}$. Choose $\mathrm{GL}_{2}(q) \leq \hat{G} \leq \Gamma L_{2}(q)$. Write $\hat{G}=\Gamma \ltimes \mathrm{GL}_{2}(q)$ with $\Gamma \leq \operatorname{Aut}\left(\mathbb{F}_{q}\right)$. Let $S$ be a proper subgroup of $\mathbb{F}_{q}^{\star}$. Set

$$
\begin{aligned}
\Delta & =\left\{\left(\begin{array}{ll}
s & 0 \\
0 & s
\end{array}\right) \mid s \in S\right\}, \\
\hat{U} & =\Gamma \ltimes\left\{\left(\begin{array}{ll}
a & c \\
0 & b
\end{array}\right) \mid a \in S, b \in \mathbb{F}_{q}^{\star}, c \in \mathbb{F}_{q}\right\}, \\
\hat{V} & =\Gamma \ltimes\left\{\left(\begin{array}{ll}
a & c \\
0 & b
\end{array}\right) \mid a \in \mathbb{F}_{q}^{\star}, b \in S, c \in \mathbb{F}_{q}\right\}, \\
G & =\hat{G} / \Delta, \\
U & =\hat{U} / \Delta, \\
V & =\hat{V} / \Delta .
\end{aligned}
$$

Lemma 2.15. $U$ and $V$ are Kronecker conjugate in $G$, but not conjugate in $G$.

Proof. For $v \in \hat{V}$ write $v=\gamma m$ with $\gamma \in \Gamma$. Then $m$ has eigenvalue $b \in S$. So $m$, and therefore $v$ as well, is conjugate to an element in $\hat{U}$. Conversely, every element in $\hat{U}$ is conjugate to some element in $\hat{V}$. From this the Kronecker conjugacy of $U$ and $V$ in $G$ follows.

Now suppose that $U$ and $V$ are even conjugate in $G$. Then, also the subgroups $\left\{\left(\begin{array}{ll}a & c \\ 0 & b\end{array}\right) \mid a \in S, b \in \mathbb{F}_{q}^{*}, c \in \mathbb{F}_{q}\right\}$ and $\left\{\left(\begin{array}{ll}a & c \\ 0 & b\end{array}\right) \mid a \in \mathbb{F}_{q}^{\star}, b \in S, c \in \mathbb{F}_{q}\right\}$ are conjugate in $\mathrm{GL}_{2}(q)$. But this is easily seen to be not the case.

For the group $G$ as defined above we will use the notation $G=\hat{G} / C_{s}$, where $s$ is the order of $S$.

Proposition 2.16. Suppose $\mathrm{GL}_{2}(q) \leq \hat{G} \leq \Gamma L_{2}(q)$, and set $G=\hat{G} / C_{s}$ for some proper subgroup $S$ of $\mathbb{F}_{q}^{\star}$ of order s. Suppose there are subgroups $U, V, M, N$ of $G$ as follows. $N$ is the core of $M$ in $G$, and $U, V$ are subgroups of prime index $p$ of $M$ such that $M=U N=V N$ and $U \cap N=V \cap N=1$. Further, assume that $U$ and $V$ are Kronecker conjugate in $G$ and that the action of $G / N$ on the cosets of $M$ in $G$ is the natural action of the corresponding group between $\mathrm{PGL}_{2}(q)$ and $\mathrm{P}_{2}(q)$.

Let $\Delta$ be as above, and for $(\lambda, \mu) \in\{(1, i), 0 \leq i \leq p-1\} \cup\{(0,1)\}$ set

$$
\hat{W}_{(\lambda, \mu)}=\Gamma \ltimes\left\{\left(\begin{array}{ll}
a & c \\
0 & b
\end{array}\right) \mid a^{\lambda} b^{\mu} \in S\right\} .
$$

Then one of the following holds.

(i) $U$ is conjugate to $\hat{W}_{(1,0)} / \Delta$ and $V$ is conjugate to $\hat{W}_{(0,1)} / \Delta$, or vice versa. 
(ii) $U$ is conjugate to $\hat{W}_{(1, \mu)} / \Delta$ and $V$ is conjugate to $\hat{W}_{\left(1, \mu^{\prime}\right)} / \Delta$ with $\mu \mu^{\prime} \equiv 1$ $(\bmod p)$.

In particular, if $p \in\{2,3\}$, then $U$ and $V$ are (up to interchanging) as in Lemma 2.15.

Proof. First one proves that a subgroup $W$ of index $p$ in $M$ such that $M=N W$ and $W \cap N=1$ has the form (up to conjugacy) of $W_{(\lambda, \mu)}$ as above. It remains to check Kronecker conjugacy of these subgroups. This is done by comparing the eigenvalues of the elements in $\hat{W}_{(\lambda, \mu)}$, where the $\Gamma$-part is omitted. The procedure is straightforward.

\section{Proof of Theorems 1.2 and 1.4 .}

Throughout this section we use standard notation from finite group theory (see [14] and [35]) and notation which has been defined in the previous sections.

3.1. Group theoretic preparation. Suppose that $f, g$ are polynomials as in Theorem 1.2 or 1.4. Write $f(X)=a(b(X))$ with indecomposable polynomials $a, b \in$ $K[X]$. Let $t$ be a transcendental number. Denote by $\Pi$ a minimal Galois extension of $K(T)$ which contains $x$ and $y$ with $f(x)=g(y)=t$. Set $G=\operatorname{Gal}(\Pi \mid K(t))$. Denote by $U$ the stabilizer of $x$ in $G$, by $V$ the stabilizer of $y$, and by $M$ the stabilizer of $b(x)$. Then $U<M<G$, and $G$ acts faithfully on the coset spaces $G / U$ and $G / V$ (see section 2.3). Set $N=\operatorname{core}_{G}(M)>1$ (see Lemma 2.6 and 2.7). The faithful action of $G / N$ on the coset space $G / M$ gives the arithmetic monodromy group of the polynomial $a$, and the induced action of $M$ on $M / U$ is the arithmetic monodromy group of $b$. Further, $U$ is maximal in $M$, and $M$ is maximal in $G$.

Now $N U$ and $N V$ are also Kronecker conjugate in $G$, and $N U=M$ as $U$ is maximal in $M$ and $N \not \subset U$. We have $V<N V<G$. Let $g(X)=c(d(X))$ be a corresponding decomposition (Lemma 2.7). Now $a$ and $c$ are Kronecker conjugate. If we are in the situation of Theorem 1.2, then $a$ and $c$ are linearly related over $K$ by Theorem 1.1, and in the situation of Theorem 1.4 again $a$ and $c$ are linearly related over $K$ by the assumption and Theorem 1.3. Thus $N V$ and $N U=M$ are conjugate by Lemma 2.10. So from now on we assume without loss that $V<M$.

Lemma 3.1. $V$ is maximal in $M$.

Proof. Let $V<W<M$. Then $C=\operatorname{core}_{G}(W)>1$, and $V<C V \leq W<M$. Now $C U=M$ and $C V$ are Kronecker conjugate in $G$. Thus, by Lemma 2.13, $|M|=|C U|=|C V|<|M|$, a contradiction.

In the following we will consider $G$ as a permutation group on the right coset space $G / U$. This action is imprimitive; a block system is provided by the right cosets $G / M$. Then $N$ is the kernel of the action of $G$ on $G / M$. Note that $M \cap Z \leq N$ (with $Z$ from Lemma 2.11). Let $N_{0} \leq N$ be a minimal normal subgroup of $G$. Set

$$
N_{U}=\operatorname{core}_{M}(U), N_{V}=\operatorname{core}_{M}(V) .
$$

Further, denote by $\widetilde{Y}$ the image of $Y \leq M$ in $M / N_{U}$. Thus $\widetilde{M}$ acts faithfully and primitively on $M / U$.

Lemma 3.2. $N_{0}$ is an elementary abelian p-group. 
Proof. The configuration is similar to that studied in [24]; thus we use some arguments from the proof of [24, Lemma 5.1]. Suppose the assertion is wrong. Then, as $N_{0}$ is a minimal normal subgroup of $G$, it can be written as $N_{0}=S_{1} S_{2} \cdots S_{t}$, the direct product of simple non-abelian groups $S_{i}$. These $S_{i}$ 's are permuted transitively by $G$. First we contend

Exactly one of the $S_{i}$ 's, say $S_{1}$, is not contained in $N_{U}$. Further, $M \leq$ $\mathbf{N}_{G}\left(S_{1}\right)$.

Since $M=(M \cap Z) U$, the group $\widetilde{M \cap Z}$ is a cyclic transitive subgroup of the primitive permutation group $\widetilde{M}$. Moreover, $\widetilde{M}$ is not solvable, as $N_{0}$ has a nontrivial image in $\widetilde{M}$; hence $\widetilde{M}$ is 2-transitive on $M / U$ by theorems of Schur and Burnside [35, Theorems 25.3 and 11.7]. Because $\widetilde{M}$ is a 2-transitive permutation group, it has a unique minimal normal subgroup $\widetilde{S}$ which is either elementary abelian or simple non-abelian (see [35, Exercise 12.4]). As $\widetilde{N_{0}} \unlhd \widetilde{M}$ is a nontrivial product of groups isomorphic to $S_{1}$, exactly one of the $S_{i}$ 's is not contained in $N_{U}$. The latter assertion follows from the former, as $M$ permutes the $S_{i}$ 's.

$$
N_{U}=\mathbf{C}_{M}\left(S_{1}\right) \text {. }
$$

$\mathbf{C}_{\widetilde{M}}\left(\widetilde{S_{1}}\right)$ is normal in $\widetilde{M}$, but does not contain $\widetilde{S_{1}}$, as $\widetilde{S_{1}}$ is not abelian. Thus $\mathbf{C}_{\widetilde{M}}\left(\widetilde{S_{1}}\right)$ is trivial. In particular, $\mathbf{C}_{M}\left(S_{1}\right) \leq N_{U}$. We get the other inclusion as follows: $S_{1}$ is simple and normal in $M$ by the previous assertion. Thus $S_{1} \cap N_{U}=1$ and therefore $N_{U} \leq \mathbf{C}_{M}\left(S_{1}\right)$.

By symmetry we also get

There is an index $i$ such that all the $S_{j}$ except for $j=i$ are contained in $N_{V}, M \leq \mathbf{N}_{G}\left(S_{i}\right)$, and $N_{V}=\mathbf{C}_{M}\left(S_{i}\right)$.

Assume for a moment that $i=1$. We then get that $\widetilde{M}$ acts faithfully and 2transitively on the coset spaces $M / U$ and $M / V$, and in both actions $\widehat{M \cap Z}$ is a transitive cyclic subgroup. But this forces $U$ and $V$ to be Kronecker conjugate; see $[6,4.1]$. But then $U$ and $V$ are even conjugate in $M$, by an argument similar to the one at the beginning of this section, a contradiction.

Observe that $Z$ permutes the $S_{i}$ 's transitively, because $G$ does so, $M$ fixes $S_{1}$, and $G=M Z$. Therefore $\mathbf{N}_{G}\left(S_{j}\right) \cap Z$ is independent of $j$. By the previous steps we know that $M$ is contained in $\mathbf{N}_{G}\left(S_{1}\right)$ and $\mathbf{N}_{G}\left(S_{i}\right)$; hence

$$
\mathbf{N}_{G}\left(S_{1}\right)=\left(\mathbf{N}_{G}\left(S_{1}\right) \cap Z\right) M=\left(\mathbf{N}_{G}\left(S_{i}\right) \cap Z\right) M=\mathbf{N}_{G}\left(S_{i}\right) .
$$

Pick $g \in G$ with $S_{i}=S_{1}^{g}$. Then

$$
\mathbf{N}_{G}\left(S_{1}\right)=\mathbf{N}_{G}\left(S_{i}\right)=\mathbf{N}_{G}\left(S_{1}\right)^{g} .
$$

Now, as $M$ is a maximal subgroup of $G$, the group $\mathbf{N}_{G}\left(S_{1}\right)$ is either $M$ or $G$. If the latter happens, then $N_{0}=S_{1}$; hence of course $i=1$, and we are done. Thus, assume $\mathbf{N}_{G}\left(S_{1}\right)=M$. If $\mathbf{N}_{G}(M)=M$, then $g$ normalizes $S_{1}$; therefore $S_{i}=S_{1}^{g}=S_{1}$, and hence again $i=1$. So it remains to check the case $M \triangleleft G$. As the normalizer of $S_{1}$ in $G$ is $M$, we get $N_{U}=\mathbf{C}_{G}\left(S_{1}\right)$ and $N_{V}=\mathbf{C}_{G}\left(S_{i}\right)$. So $N_{U}^{g}=N_{V}$, and both $N_{U}^{g}$ and $N_{V}$ are contained in $M$. As in the case $N_{U}=N_{V}$, we get that $U^{g}$ and $V$ are conjugate in $M$, a contradiction.

In virtue of the previous lemma, we now suppose that $N_{0}$ is elementary abelian of exponent $p$ for some prime $p$.

Lemma 3.3. One of the following holds. 
(a) $[M: U]=[M: V]=p$ and $C_{p} \leq M / N_{U}, M / N_{V} \leq \operatorname{AGL}_{1}(p)$.

(b) $[M: U]=[M: V]=4$ and $M / N_{U}=M / N_{V}=\mathrm{S}_{4}$.

Proof. The group $\widetilde{N_{0}}$ is an elementary abelian normal and transitive $p$-subgroup of $\widetilde{M}$. Set $\left|N_{0}\right|=p^{k}$. Then $\widetilde{M}$ imbeds into $\operatorname{AGL}_{k}(p)$, with the latter group acting naturally on $\mathbb{F}_{p}^{k}$. Now $\operatorname{AGL}_{k}(p)$ is a subgroup of $\operatorname{GL}_{k+1}(p)$, and as $\widetilde{M}$ contains the cyclic subgroup $\widetilde{M \cap Z}$ of order $p^{k}$, we infer that $\mathrm{GL}_{k+1}(p)$ contains an element of order $p^{k}$. This easily forces $k=1$ or $p^{k}=4$. From this the assertion follows quickly.

Note that in case (a) nothing guarantees that $M / N_{U}$ and $M / N_{V}$ are isomorphic.

Lemma 3.4. Neither $N_{U} \leq N_{V}$, nor $N_{V} \leq N_{U}$.

Proof. In a group between $C_{p}$ and $\mathrm{AGL}_{1}(p)$, any two subgroups of index $p$ are conjugate (for instance by Schur-Zassenhaus). Also, in $\mathrm{S}_{4}$ any two subgroups of index 4 are conjugate.

We need some more notation. Let $\Omega$ be the coset space $G / U$. If not otherwise said, $G$ is considered as a permutation group on $\Omega$. The cosets of $M$ in $G$ provide a system of imprimitivity for the action of $G$. Denote these blocks by $\Omega_{1}, \Omega_{2}, \ldots, \Omega_{m}$, where $\Omega_{1}$ should be the coset $M$. That is, $M$ fixes $\Omega_{1}$. Let $\bar{\Omega}$ be the set $\left\{\Omega_{1}, \Omega_{2}, \ldots, \Omega_{m}\right\}$. For a subgroup $Y$ of $G$, set $\bar{Y}=Y N / N$. Then $\bar{G}$ acts faithfully on $\bar{\Omega}$. Set $\bar{\Omega}^{*}=\bar{\Omega} \backslash \Omega_{1}$. So $\bar{M}$ acts faithfully on $\bar{\Omega}^{*}$.

Further, we set $Z_{p}=M \cap Z$. Note that $\left|Z_{p}\right|=p$, and that $Z_{p}$ acts regularly on each $\Omega_{i}$.

Lemma 3.5. Every element of $N_{U} \backslash N_{V}$ fixes an element of $\bar{\Omega}^{*}$.

Proof. Let $u \in N_{U}$. Pick an arbitrary $m \in Z_{p}$. From $M=V Z_{p}$ we get $M=V^{m} Z_{p}$. Thus $u=v^{m} z$ for some $v \in V, z \in Z_{p}$. By Kronecker conjugacy, $v$ fixes some element in $\Omega$, so in particular it fixes some element in $\bar{\Omega}$. As $m, z \in Z_{p} \subseteq N$, the elements $v^{m} z$ and $v$ fix the same elements in $\bar{\Omega}$. Now suppose that $u=v^{m} z$ fixes only the set $\Omega_{1}$. Then $v$, and then also $v^{m}$, fixes an element in $\Omega_{1}$. But $v^{m} z=u$ is trivial on $\Omega_{1}$ (as $u \in N_{U}$ ); therefore $z=1$. Thus $v^{m}=u$. As $m$ was arbitrarily chosen, we get $u \in \bigcap_{m \in Z_{p}} V^{m}=\bigcap_{m \in M} V^{m}=N_{V}$, and the assertion follows.

Lemma 3.6. If $M / N_{U}=\mathrm{S}_{4}$, then $N$ acts as $\mathrm{S}_{4}$ on each block $\Omega_{i}$.

Proof. The group $N_{U} N / N_{U}$ is normal in $M / N_{U}=\mathrm{S}_{4}$ and contains the cyclic group $Z_{4} N_{U} / N_{U}$ of order 4 . The only normal subgroup of $\mathrm{S}_{4}$ containing such a group is $\mathrm{S}_{4}$ itself. Thus $N_{U} N / N_{U}=\mathrm{S}_{4}$.

The list in 2.8 tells us that either $\bar{G}$ is 3 -transitive and distinct from $\mathrm{S}_{3}$, or $C_{r} \leq \bar{G} \leq \mathrm{AGL}_{1}(r)$ for some prime $r$. These two cases require totally different arguments, so we treat them in separate subsections.

3.2. The case when $\bar{G} \neq \mathrm{S}_{3}$ is 3 -transitive. We assume throughout this subsection that $\bar{G}$ is 3 -transitive and different from $\mathrm{S}_{3}$. Then $\bar{M}$ is 2 -transitive on $\bar{\Omega}^{*}$, so every normal subgroup of $\bar{M}$ is either trivial or transitive on $\bar{\Omega}^{*}$.

Lemma 3.7. $N_{U} \cap N_{V} \not \leq N$. 
Proof. Suppose not; then $\overline{N_{U} \cap N_{V}}=1$. So $\overline{N_{U}}$ is intransitive on $\bar{\Omega}^{*}$ by 3.5 (as every transitive permutation group has fixed point free elements); thus even $N_{U} \leq N$. By symmetry we also get $N_{V} \leq N$. Thus $N_{U} N_{V} \leq N$. Therefore $\bar{M}$ is a homomorphic image of $M / N_{U} N_{V}$. We get the sequence of surjections

$$
U / N_{U} \rightarrow U N_{V} / N_{U} N_{V}=M / N_{U} N_{V} \rightarrow \bar{M} .
$$

If we are in case (a) of Lemma 3.3, then $U / N_{U}$ is a subgroup of the cyclic group of order $p-1$, so 2 -transitivity of $\bar{M}$ forces $\bar{M}=C_{2}$, and so $\bar{G}=\mathrm{S}_{3}$, a case ruled out from consideration here.

Now suppose that we are in case (b) of Lemma 3.3. Then $M=N_{U} N$ by 3.6. But we got $N_{U} \leq N$, so $M=N$, and thus $\bar{M}=1$, a contradiction.

Lemma 3.8. $U \cap N=V \cap N \leq N_{U} \cap N_{V}$.

Proof. By 3.7 the group $\overline{N_{U} \cap N_{V}}$ is transitive on $\bar{\Omega}^{*}$. Thus there is $x \in N_{U} \cap N_{V}$ such that $x$ has no fixed point on $\bar{\Omega}^{*}$. Each element in $x \cdot(V \cap N) \subseteq V$ has, by Kronecker conjugacy, a fixed point in $\Omega$, and this point therefore has to lie in $\Omega_{1}$, because all the other blocks $\Omega_{i}$ are moved. As $x$ is trivial on $\Omega_{1}$, every element in $V \cap N$ has a fixed point on $\Omega_{1}$. But $\widetilde{V \cap N}$ is normal in the primitive (on $\Omega_{1}$ ) group $\widetilde{V}$, so is must be trivial. So $V \cap N \leq N_{U}$, in particular $V \cap N \leq U \cap N$, and the rest follows by symmetry.

The next assertion rids us of the case $M / N_{U}=\mathrm{S}_{4}$.

Lemma 3.9. $[M: U]=[M: V]=p$ and $C_{p} \leq M / N_{U}, M / N_{V} \leq \mathrm{AGL}_{1}(p)$.

Proof. Suppose $M / N_{U}=\mathrm{S}_{4}$. Then $M=N_{U} N$ by 3.6. Thus $U=N_{U}(U \cap N)$. From 3.8 we further get $U \leq N_{U}$, which of course is nonsense.

So from now on $p \in \mathbb{P}$. We introduce some more notation. Set

$$
\begin{aligned}
& A=N_{U} \cap N_{V} Z_{p}, \\
& B=N_{U} \cap N_{V} .
\end{aligned}
$$

Note that $A$ and $B$ are normal subgroups of $M$.

Lemma 3.10. $|A / B|=p$.

Proof. $N_{V} Z_{p} / N_{V}$ is the unique minimal normal subgroup of $M / N_{V}$; thus

$$
N_{V} Z_{p} \leq N_{U} N_{V}
$$

Therefore the natural map

$$
A \longrightarrow N_{V} Z_{p} / N_{V}
$$

is surjective with kernel $B$, and the assertion follows.

Here, and in the following, we need the easy

Proposition 3.11. Let $X$ be a (not necessarily faithful) transitive permutation group with a subgroup $Y$, such that every element in $X \backslash Y$ has a fixed point. Then every element in $X \backslash Y$ has exactly one fixed point. 
Proof. Let $o$ be the number of orbits of $Y$. Denote by $F(x)$ the number of fixed points of $x$. The orbit formula yields

$$
\begin{aligned}
1 & =\frac{1}{|X|}\left(\sum_{x \in X \backslash Y} F(x)+\sum_{y \in Y} F(y)\right) \\
& \geq \frac{1}{|X|}((|X|-|Y|)+|Y| o),
\end{aligned}
$$

and hence $o \leq 1$. Thus $o=1$, and equality holds everywhere.

Lemma 3.12. $U \cap N=V \cap N=1$, and $N=Z_{p}$.

Proof. From 3.7 we know that $B$ is not contained in $N$. Thus $A$ is transitive on $\bar{\Omega}^{*}$. Choose $a \in A \backslash B$. By 3.5 and 3.11 we get that $a$ fixes exactly one element in $\bar{\Omega}^{*}$. Write $a=z v$ with $z \in Z_{p}$ and $v \in N_{V}$. Then $z \neq 1$, because $a \notin N_{V}$. As $a$, and hence $v$, fixes exactly one element $\Omega_{i}$ in $\bar{\Omega}^{*}$, and $v$ has order $p$ on $\Omega_{1}, v$ fixes a point in $\Omega_{i}$. Set $w=v^{p-1}$. By $3.10, a^{p-1}$ is still in $A \backslash B$, so $a^{p-1}$ and thus also $w$ fixes exactly one element in $\bar{\Omega}^{*}$. As $v$ has order $p$ on $M$, and order a divisor of $p-1$ on $\Omega_{i}$, we conclude that $w$ is trivial on $\Omega_{i}$, non-trivial on $\Omega_{1}$, and moves all the blocks $\Omega_{j}$ except for $\Omega_{1}$ and $\Omega_{i}$. By 3.8 we have $V \cap N \leq N_{U}$, so every element in $w(V \cap N)$ has fixed points only on $\Omega_{i}$, and the same holds for $V \cap N$. But $V \cap N$ acts as a subgroup of $\operatorname{AGL}_{1}(p)$ on $\Omega_{i}$, so it fixes a point in $\Omega_{i}$. Thus $V \cap N \leq U^{t} \cap N=(U \cap N)^{t}$ for some $t \in G \backslash M$. Again using 3.8 we get $V \cap N \leq N_{U}^{t}$. The $\Omega_{i}$ we got could have been any $\Omega_{j}$ different from $\Omega_{1}$ (by transitivity of $A$ on $\left.\bar{\Omega}^{*}\right)$, so there is a set of coset representatives $\left\{t_{1}, t_{2}, \ldots, t_{m}\right\}$ of $M$ in $G$ such that $V \cap N \leq N_{U}^{t_{i}}$ for all $i$. As $N_{U} \unlhd M$, we get $V \cap N \leq \operatorname{core}_{G}\left(N_{U}\right)=1$. The latter assertion is obvious, as $Z_{p} \leq N$ has regular orbits on each $\Omega_{i}$.

Lemma 3.13. $M / N_{U}$ is a homomorphic image of $\bar{M}=M / N$.

Proof. By 3.12 we get

$$
M / N=V N / N \cong V \rightarrow V N_{U} / N_{U}=M / N_{U} .
$$

The next proposition collects the information to be used most frequently in the following.

Proposition 3.14. The following hold.

(a) $[M: U]=[M: V]=p$ and $C_{p} \leq M / N_{U}, M / N_{V} \leq \mathrm{AGL}_{1}(p)$ for some prime $p$.

(b) $|\bar{A} / \bar{B}|=p$.

(c) $\left|\bar{M} / \overline{N_{U}}\right|$ divides $p-1$.

(d) $\left|\overline{N_{U}} / \bar{B}\right|$ divides $p(p-1)$.

(e) Every element of $\bar{A} \backslash \bar{B}$ fixes an element in $\bar{\Omega}^{*}$.

(f) $N=Z_{p}$.

(g) $M / N_{U}$ is a homomorphic image of $M / N=\bar{M}$.

Proof. (a) is just 3.9 .

(b) follows from 3.12, as $A$ has trivial intersection with $N$.

As for (c), we have (as $N=Z_{p}$ by 3.12) $\bar{M} / \overline{N_{U}}=M Z_{p} / N_{U} Z_{p}=M / N_{U} Z_{p}$, and the assertion follows from (a). 
(d) follows from $N_{U} \cap N=1$ (3.12) and $N_{U} / B=N_{U} / N_{U} \cap N_{V}=N_{U} N_{V} / N_{V} \leq$ $M / N_{V}$.

(e) follows from 3.5, (f) is 3.12 , and (g) is 3.13 .

The strategy now is the following. The list in 2.8 tells us that $\bar{G}$ is one of the following groups:

$\mathrm{A}_{m}(m \geq 5$ odd $), \mathrm{S}_{m}(m \geq 4), \mathrm{M}_{11}, \mathrm{M}_{23}, \mathrm{PGL}_{2}(5), \mathrm{PGL}_{2}(7), \mathrm{P}_{2}(8)$, and $\mathrm{P}_{2}(9)$.

In $\bar{M}$ we find the normal subgroups $\bar{B} \triangleleft \bar{A} \unlhd \overline{N_{U}}$, and use the information provided in Proposition 3.14 to determine the possibilities for $G / N$ and $N$ and the action of $G / N$ on $N$. If $N$ has a complement in $G$, we can immediately write down $G$. In the other cases, we prefer to use the classification of transitive groups of small degrees, or in two instances the computer algebra system GAP (see [30]) to determine the possibilities.

Lemma 3.15. The extension

$$
1 \rightarrow N \rightarrow M \rightarrow M / N \rightarrow 1
$$

splits. Let $m=[G: M]$ be the degree of $\bar{G}$. If $(m, p)=1$, then also

$$
1 \rightarrow N \rightarrow G \rightarrow G / N \rightarrow 1
$$

splits.

Proof. The first part follows from 3.12, as $U$ is a complement of $N$ in $M$.

Let $f \in \mathrm{H}^{2}(G / N, N)$ describe the extension $1 \rightarrow N \rightarrow G \rightarrow G / N \rightarrow 1$. Then, by the first part,

$$
\operatorname{res}_{G / N \rightarrow M / N}(f)=0 .
$$

On the other hand, we have the well-known relation between restriction and corestriction of cohomology groups

$$
\operatorname{cor}_{M / N \rightarrow G / N}\left(\operatorname{res}_{M / N \rightarrow G / N}(x)\right)=x^{m}
$$

for all $x \in \mathrm{H}^{2}(G / N, N)$. As $(m,|N|)=1$, the restriction is injective, so $f=0$ and the assertion follows.

Lemma 3.16. If $\bar{G}=\mathrm{A}_{m}$, then $m=5, p=3$, and $G=\mathrm{GL}_{2}(4)$.

Proof. By $3.14 \bar{M}$ has normal subgroups $\bar{A}$ and $\bar{B}$ with $|\bar{A} / \bar{B}|=p$. If $m \geq 6$, then $\bar{M}=\mathrm{A}_{m-1}$ is simple, so this cannot occur at all. If $m=5$, then $\bar{M}=\overline{N_{U}}=\bar{A}=\mathrm{A}_{4}$, $\bar{B}=C_{2} \times C_{2}$ is the only configuration such that each element in $\bar{A} \backslash \bar{B}$ has a fixed point.

Now $N=C_{3}$ (by $3.14(\mathrm{f})$ ), and $\bar{G}=\mathrm{A}_{5}$ can act only trivially on $C_{3}$. From 3.15 we gets $G=\mathrm{GL}_{2}(4)$.

Lemma 3.17. If $\bar{G}=\mathrm{S}_{m}$, then either $m=5, p=3$, and $G=\Gamma \mathrm{L}_{2}(4)$, or $m=4$, $p=2$, and $G=\mathrm{GL}_{2}(3)$.

Proof. If $m \geq 6$, then the only possibility could be $\bar{A}=\mathrm{S}_{m-1}, \bar{B}=\mathrm{A}_{m-1}$. But it is immediate that for $m \geq 6$ there are fixed-point-free elements in $S_{m-1} \backslash A_{m-1}$; for instance if $m-1$ is odd, then choose an $(m-1)$-cycle, and if $m-1$ is even, then take the product of an $(m-3)$-cycle with a disjoint transposition.

Thus $m \leq 5$. If $m=5$, then as in 3.16 we get $\overline{N_{U}}=\bar{A}=\mathrm{A}_{4}, \bar{B}=C_{2} \times C_{2}$; thus $p=3$. By $3.14(\mathrm{~g})$ we get that $M / N_{U}$ is a homomorphic image of $\mathrm{S}_{4}$. On the other 
hand, $C_{3} \leq M / N_{U} \leq D_{3}$. That is only possible if $M / N_{U}=D_{3}$. Thus $N$ is not central in $G$. There is a unique nontrivial action of $\mathrm{S}_{4}$ on $C_{3}$, so from 3.15 we get $G=\Gamma \mathrm{L}(2,4)$.

If $m=4$, then $\bar{M}=\overline{N_{U}}=\bar{A}=\mathrm{S}_{3}$ and $\bar{B}=C_{3}$, hence $p=2$. Here $\mathrm{H}^{2}(G / N, N)=C_{2} \times C_{2}$, so rather than considering the 4 cases we use the classification of transitive groups of small degree [2]: There are just two groups of degree 8 and size 48 . One is $\mathrm{GL}_{2}(3)$ and the other one is $\mathrm{S}_{2} \times \mathrm{S}_{4}$, acting on a $2 \times 4$ array with $\mathrm{S}_{2}$ switching the rows, and $\mathrm{S}_{4}$ permuting the columns. This group, however, does not contain a cyclic subgroup $Z$ of order 8 . Thus $G=\mathrm{GL}_{2}(3)$.

Lemma 3.18. $\bar{G}$ is different from $\mathrm{M}_{11}$ and $\mathrm{M}_{23}$.

Proof. The case $\bar{G}=\mathrm{M}_{23}$ dies immediately, as $\bar{M}=\mathrm{M}_{22}$ is simple, and so the required normal subgroup $\bar{B}$ does not exist.

Now suppose $\bar{G}=\mathrm{M}_{11}$. Then the simple group $\mathrm{PSL}_{2}(9)$ has index 2 in $\bar{M}$. Thus $\bar{A}=\bar{M}$ and $\bar{B}=\mathrm{PSL}_{2}(9)$. As $\mathrm{M}_{11}$ is sharply 4-transitive, $\bar{A}$ is sharply 3 -transitive. By 3.11 every element of $\bar{A}$ with exactly two fixed points lies in $\bar{B}$. The permutation group $\mathrm{PGL}_{2}(9)$ is also sharply 3 -transitive, so it must have the same number of elements with exactly two fixed points as $\bar{A}$ has, so all these elements are lying in $\mathrm{PSL}_{2}(9)$. However, that is not the case. Let $\alpha$ be a generator of the multiplicative group of the finite field $\mathbb{F}_{9}$. Then the image in $\mathrm{PGL}_{2}(9)$ of the diagonal matrix with entries 1 and $\alpha$ fixes two points, but does not lie in $\mathrm{PSL}_{2}(9)$.

Lemma 3.19. If $\bar{G}=\mathrm{PGL}_{2}(5)$, then $p=2$ and $G=\mathrm{GL}_{2}(5) / C_{2}$ (see section 2.4 for this notation).

Proof. We have $C_{5} \leq \bar{B}<\bar{A} \leq \overline{N_{U}} \leq \bar{M}=\mathrm{AGL}_{1}(5)$. Thus $p=2$ by $3.14(\mathrm{~g})$. A look at [28] shows that $\mathrm{GL}_{2}(5) / C_{2}$ and $\mathrm{S}_{2} \times \mathrm{PGL}_{2}(5)$ are the only transitive groups of degree 12 and size 240 . As $G$ contains an element of order 12 , only $G=\mathrm{GL}_{2}(5) / C_{2}$ is possible.

Lemma 3.20. If $\bar{G}=\mathrm{PGL}_{2}(7)$, then $p=2, G=\mathrm{GL}_{2}(7) / C_{3}$, or $p=3, G=$ $\mathrm{GL}_{2}(7) / C_{2}$.

Proof. As in the previous case we get $p=2$ or 3 . First consider $p=3$. As $\mathrm{AGL}_{1}(7) \rightarrow M / N_{U}(3.14(\mathrm{f}))$, we have $M / N_{U} \leq C_{6}$, so $M / N_{U}=C_{3}$. Therefore $G$ is a subgroup of the wreath product $C_{3} 2 \mathrm{PGL}_{2}(7)$; in particular, $N$ is central in $G$. From 3.15 we get $G=\mathrm{GL}_{2}(7) / C_{2}$.

The case $p=2$ is more elaborate. The second cohomology $\mathrm{H}^{2}(G / N, N)$ has size four; also there is no list of the transitive groups of degree 16 . So we prefer to make an exhaustive search as follows. $\mathrm{PGL}_{2}(7)$ is generated by the 8 -cycle $\left(\begin{array}{llll}1 & 2 & 3 & \cdots\end{array}\right)$ and (1 7)(2 8)(3 4). Set $a_{i}=(i i+8)$ for $i=1,2, \ldots, 8$ and $W:=\left\langle a_{1}, a_{2}, \ldots, a_{8}\right\rangle$. Set $\sigma_{1}=(123 \cdots 8)(91011 \cdots 16), \sigma_{2}:=(17)(28)(34)(915)(1016)(1112)$.

Then $G \leq C_{2}\left\langle\mathrm{PGL}_{2}(7)=\left\langle W, \sigma_{1}, \sigma_{2}\right\rangle\right.$. Then $N$ is generated by $\sigma_{3}:=a_{1} a_{2} \cdots a_{8}$. There are $w_{1}, w_{2} \in W$ with $G=\left\langle w_{1} \sigma_{1}, w_{2} \sigma_{2}, \sigma_{3}\right\rangle$.

To find the possibilities for $w_{1}, w_{2}$, first note that we may replace $w_{1} \sigma_{1}$ by the conjugate $\left(w_{1} \sigma_{1}\right)^{w}$ for $w \in W$; this amounts of switching the labellings of certain pairs $i, i+8$. As $\sigma_{1}$ is an 8 -cycle, a moment's thought shows that there are just two orbits under the action of $W$ on $W \sigma_{1}$ by conjugation; they are given by (19) $\sigma_{1}$ and $\sigma_{1}$. For $w_{2}$ we have to consider all the $2^{8}$ possibilities, though, and to check when $\left|\left\langle w_{1} \sigma_{1}, w_{2} \sigma_{2}, \sigma_{3}\right\rangle\right|=2 \cdot\left|\mathrm{PGL}_{2}(7)\right|$. Then we check the surviving groups for conjugacy in the full wreath product $C_{2} \prec \mathrm{PGL}_{2}(7)$, and find that there are just the 
two cases, $\mathrm{GL}_{2}(7) / C_{3}$ and $\mathrm{S}_{2} \times \mathrm{PGL}_{2}(7)$. As in the former cases, we conclude that $G=\mathrm{GL}_{2}(7) / C_{3}$. The implementation of the procedure just described in GAP is straightforward, and so we omit it here.

Lemma 3.21. $\bar{G}$ is different from $\mathrm{P}_{2}(8)$.

Proof. The argument as given in 3.19 shows that $p=3$ or $p=7$. Suppose $p=3$. Then $\left|\bar{M} / \overline{N_{U}}\right|$ divides $p-1=2$ (by 3.14(c)), and it also divides $\left[\mathrm{A \Gamma L}_{1}(8): C_{8}\right]=$ $3 \cdot 7$, so $\bar{M}=\overline{N_{U}}$.

By 3.14(b) and 3.14(d) we also get that $\left|\overline{N_{U}} / \bar{A}\right|$ divides $p-1$ (and also $3 \cdot 7$ ); hence $\bar{A}=\bar{M}=\mathrm{A}_{1}(8)$, and therefore $\bar{B}=\mathrm{AGL}_{1}(8)$. It is easy to see that each element in $\mathrm{A \Gamma L}_{1}(8) \backslash \mathrm{AGL}_{1}(8)$ has no or exactly two fixed points; thus, by 3.11 , each such element is contained in $\bar{B}$, a contradiction.

To rule out $p=7$ we have to recall the polynomial context our groups came from, for otherwise $G=\Gamma L_{2}(8)$, for instance, would be an example matching all the group theoretic requirements used so far.

Let $\dot{G}$ be the geometric monodromy group of the polynomial $f$; then $\dot{G}$ is a transitive normal subgroup of $G$ (see section 2.2). For $X \leq G$ set $\dot{X}=\dot{G} \cap X$. We still have $N=Z_{2} \leq \dot{G}$. As no nontrivial normal subgroup of $\operatorname{P} L_{2}(8)=G / N$ is a monodromy group of a polynomial (see Proposition 2.8), we get $G=\dot{G} N$; thus $G=\dot{G}$. So we may assume that $G$ is already a geometric monodromy group. From $3.14(\mathrm{~g})$ we get $\mathrm{A} L_{1}(8) \rightarrow M / N_{U}$; thus $M / N_{U}=C_{7} \rtimes C_{3}$. So also $C_{7} \rtimes C_{3}$ is a geometric monodromy group of a polynomial, contrary to Proposition 2.8.

Lemma 3.22. If $\bar{G}=\mathrm{P} \mathrm{L}_{2}(9)$, then $p=2$ and $G=\Gamma \mathrm{L}_{2}(9) / C_{4}$.

Proof. We have $\left[\mathrm{A \Gamma L}_{1}(9): C_{9}\right]=2^{3} \cdot 2$; hence $p=2$ as in 3.19. To exhibit the structure of $G$, we use the analogous procedure as in the case $\bar{G}=\mathrm{PGL}_{2}(7)$, $p=2$.

3.3. The case $C_{r} \leq \bar{G} \leq \operatorname{AGL}_{1}(r)$. We are left to consider the case that $C_{r} \leq \bar{G} \leq$ $\mathrm{AGL}_{1}(r)$ for some prime $r$. In order to rule out many group theoretic configurations (which otherwise could occur), we will partially make use of the genus 0 condition (see section 2.2) or argue directly with the polynomials $f(X)=a(b(X)), g(X)=$ $a\left(b^{\prime}(X)\right)$. As in the proof of Lemma 3.21, we will thus use the geometric monodromy group $\dot{G} \unlhd G$. For $X \leq G$ set $\dot{X}:=\dot{G} \cap X$. We use the information about the genus 0 generators of $\dot{G}$ as in 2.2 without further comment. The first lemma already shows that this case behaves completely different from the case that $\bar{G}$ is triply transitive (compare with Lemma 3.7).

Lemma 3.23. $N_{U} N_{V} \leq N$.

Proof. Choose $u \in N_{U} \backslash N_{V}$. Then, by Lemma 3.5, $u$ has a fixed point on $\bar{\Omega}^{*}$. On the other hand, $\bar{M}$ acts fixed-point-freely on $\bar{\Omega}^{*}$; hence $u \in N$. Thus $N_{U}=$ $\left\langle N_{U} \backslash N_{V}\right\rangle \leq N$. From symmetry we get $N_{V} \leq N$ as well.

We call a polynomial cyclic or dihedral, if it equals $X^{n}$ or $T_{n}(X)$ up to linear changes respectively. By Lemma 2.9 we know that the polynomial $a$ corresponding to $\bar{G}$ is either a cyclic or a dihedral polynomial. Let $r \in \mathbb{P}$ be its degree. In the following we will encounter two cases where $Z$ will happen to be normal in $G$. A short number theoretic argument rules this out.

Lemma 3.24. $Z$ is not normal in $G$. 
Proof. Suppose $Z \unlhd G$. The case $M / N_{U}=\mathrm{S}_{4}$ does not occur, for otherwise also $Z_{4}$ would be normal in $G$; but $Z_{4} N_{U} / N_{U}$ is not normal in $M / N_{U}$.

So $p \in \mathbb{P}$, and $G$ is a subgroup of $\mathrm{AGL}_{1}(p r)$. Set $R=\mathbb{Z} /$ pr $\mathbb{Z}$. We identify the action of $G$ on $\Omega$ with the natural action of $G$ on $R$, where $G$ consists of permutations $x \mapsto \mu x+\theta$. For elements in $R$, a congruence modulo $p$ or $r$ has its obvious meaning.

The multiplicative group of $R$ is generated by (at most) two elements, so also $U$ is generated by two elements. As $V \cong U$ (from $V \cong V Z / Z=U Z / Z \cong U$ ), also $V$ is generated by two elements. Without loss (and by Kronecker conjugacy), one of the generators fixes 0 . Let $\gamma$ be a fixed point of the other generator. Thus $V$ is generated by elements

$$
\begin{aligned}
& A: x \mapsto \alpha x, \\
& B: x \mapsto \beta x+\gamma(1-\beta) .
\end{aligned}
$$

As $V$ is abelian, we have $A B=B A$, which gives

$$
(1-\alpha)(1-\beta) \gamma=0 \text {. }
$$

As $\gamma \neq 0$ (for otherwise $V$ fixes 0 , and we are done), we assume without loss that

$$
\alpha \equiv 1 \quad(\bmod p) .
$$

The fact that $A^{e} B^{f} \in V$ has a fixed point translates to the divisibility relation $\left(\alpha^{e} \beta^{f}-1\right) \mid\left(\gamma\left(1-\beta^{f}\right)\right)$. As $\beta$ is a unit in $R$, we find an exponent $f$ such that $\alpha \beta^{f}-1 \equiv 0(\bmod r)$. So (choosing $\left.e=1\right) \alpha \beta^{f}-1$ is divisible by $r$, and divides $\gamma\left(1-\beta^{f}\right)$. But $r$ does not divide $1-\beta^{f}$, for otherwise $r$ would also divide $\left(\alpha \beta^{f}-\right.$ $1)+\left(1-\beta^{f}\right)=(\alpha-1) \beta^{f}$, so even $\alpha \equiv 1(\bmod r)$; hence $\alpha=1$, a contradiction.

So $r$ divides $\gamma$. But then $\gamma$ is also a fixed point of $A$, as $\alpha \equiv 1(\bmod r)$, so $\alpha \gamma=\gamma$. So $V$ fixes $\gamma$; that is, $U$ and $V$ are conjugate in $G$.

We are now going to rule out the case that $a$ is a dihedral polynomial.

Lemma 3.25. The polynomial a is cyclic.

Proof. Suppose otherwise. Then $a$ is a dihedral polynomial of degree $r \geq 3$. Let $\sigma_{1}, \sigma_{1}, \ldots, \sigma_{R}$ be a genus 0 system of $\dot{G}$. Without loss (using braiding), we may assume that $\overline{\sigma_{1}}, \overline{\sigma_{2}}$ is a genus 0 system corresponding to $a$, thus generating $\bar{G}$. We know that $\overline{\sigma_{1}}$ and $\overline{\sigma_{2}}$ are involutions, each fixing exactly one block $\Omega_{i}$. So suppose that $\sigma_{1}$ and $\sigma_{2}$ fix $\Omega_{i_{1}}$ and $\Omega_{i_{2}}$ setwise, respectively. Let $b_{1}$ and $b_{2}$ be the branch points of $a$ corresponding to $\overline{\sigma_{1}}$ and $\overline{\sigma_{2}}$ respectively. The preimage $a^{-1}\left(b_{i}\right)$ contains $(r-1) / 2$ double points and a single point $c_{i}$. The fiber $b^{-1}\left(c_{1}\right)$ tells us how $\sigma_{1}$ acts on $\Omega_{i_{1}}$. If all the elements in $b^{-1}\left(c_{1}\right)$ are distinct, then $\sigma_{1}$ is trivial on $\Omega_{i_{1}}$. But this cannot happen by Lemma 3.24, as then $\sigma_{1}$ is conjugate to an element in $N_{U}$, which acts as an involution on $\bar{\Omega}^{*}$, hence is not contained in $N$.

So $c_{1}$ is a branch point of the polynomial $b$, and with the same argument $c_{2}$ is too. So $b$ has at least two finite branch points, so it either is dihedral or has monodromy group $\mathrm{S}_{4}$. The latter cannot happen. Namely by Lemma 3.6 we find an $n \in N$ such that $\sigma_{1} n$ is trivial on $\Omega_{i_{1}}$, again contradicting Lemma 3.23.

So $b$ is a dihedral polynomial. By linear changes we may assume that $f(X)=$ $\rho_{1} \dot{f}\left(\rho_{3}(X)+\rho_{4}\right)+\rho_{2}$ with $\dot{f}(X)=T_{r}\left(\gamma T_{p}(X)+\delta\right)$ with $\rho_{i}, \gamma, \delta \in \mathbb{C}$ and $\rho_{1}, \rho_{3}, \gamma$ nonzero. The branch points of $T_{p}$ and $T_{r}$ are 2 and -2 . So, by the above consideration, we must have $\left\{c_{1}, c_{2}\right\}=\{2,-2\}$. This shows that either $2 \gamma+\delta=2$ and 
$-2 \gamma+\delta=-2$, or $-2 \gamma+\delta=2$ and $2 \gamma+\delta=-2$. Thus $\delta=0$ and $\gamma=1$ or -1 . As $T_{r}$ is an odd polynomial, we have $\dot{f}(X)= \pm T_{r}\left(T_{p}(X)\right)= \pm T_{r p}(X)$.

Thus $\dot{G}=D_{r p}$. But then $G$ normalizes $Z$, for if some $g \in G$ did not, then $g$ would map a generator of $Z$ to an involution in $\dot{G}$, which of course is nonsense. We are done by Lemma 3.24 .

Lemma 3.26. The polynomial $f$ has at least 2 finite branch points.

Proof. Suppose otherwise. Then $f$ is a cyclic polynomial, so $C_{r p} \leq G \leq \mathrm{AGL}_{1}(r p)$. The claim follows from Lemma 3.24.

Now again let $\sigma_{1}, \sigma_{2}, \ldots, \sigma_{R}$ be a genus 0 system for $\dot{G}$, where $\overline{\sigma_{1}}$ should generate the cyclic group $\dot{\dot{G}}$. Thus $\sigma_{2}, \sigma_{3}, \ldots, \sigma_{R}$ are in $\dot{N}$. We will use these elements to show that the group $\dot{N}$ is not too small.

Lemma 3.27. If $p \neq 4$ (that is, $M / N_{U} \neq \mathrm{S}_{4}$ ), then $C_{p}^{r} \leq \dot{N}$.

Proof. We view $G$ in the natural way as being a subgroup of the wreath product $\operatorname{AGL}_{1}(r) \ltimes \operatorname{AGL}_{1}(p)^{r}$. Write

$$
\sigma_{1}=c \cdot\left(d_{1}, d_{2}, \ldots, d_{r}\right),
$$

where $c \in \mathrm{AGL}_{1}(r)$ induces the cyclic shift $(1,2, \ldots, r)$ on the blocks $\Omega_{i}$, and with $d_{i} \in \operatorname{AGL}_{1}(p)$. We have the genus 0 condition

$$
\sum_{i=1}^{R} \operatorname{ind} \sigma_{i}=p r-1 .
$$

Next compute that

$$
\sigma_{1}^{r}=\left(\delta_{1}, \delta_{2}, \ldots, \delta_{r}\right)
$$

with $\delta_{1}=d_{1} d_{2} \cdots d_{r}$ and $\delta_{i}$ conjugate to $\delta_{1}$ in $\operatorname{AGL}_{1}(p)$. Suppose that $\delta_{1}$ has $k$ orbits on $\Omega_{1}$. Then also $\sigma_{1}$ has $k$ orbits on $\Omega$. So ind $\sigma_{1}=p r-k$. The above relation gives

$$
\sum_{i=2}^{R} \text { ind } \sigma_{i}=k-1 \leq p-1 .
$$

Suppose that one of the $\sigma_{2}, \ldots, \sigma_{R}$, say $\sigma_{w}$, induces the action of an element in $\operatorname{AGL}_{1}(p) \backslash C_{p}$ on $\Omega_{i}$ for some $i$, but fixes all the other $\Omega_{j}$ 's pointwise. Then the group generated by the commutators $\left[n, \sigma_{w}\right], n \in \dot{N}$, induces a cyclic group of order $p$ on $\Omega_{i}$, and is trivial on all the other $\Omega_{j}$ 's. Conjugating by powers of $\sigma_{1}$ then yields the assertion in this case.

Another case is that one of the elements $\sigma_{2}, \ldots, \sigma_{R}$ is trivial on all but one $\Omega_{i}$, and is a $p$-cycle on this block. Then again $C_{p}^{r} \leq \dot{N}$ as above.

Any non-trivial element in $\mathrm{AGL}_{1}(p)$ is either a $p$-cycle, hence has index $p-1$, or is an involution, hence has index $(p-1) / 2$, or has index $>(p-1) / 2$. So the only case not covered yet and which is allowed by the above genus relation is $k=p$, $R=2$, and $\sigma_{2}$ induces an involution on two $\Omega_{i}$ 's, and is trivial on all the other 
$\Omega_{j}$ 's. Then $\delta_{1}=1$. Set

$$
\begin{aligned}
\omega_{1} & =d_{2} d_{3} \cdots d_{r}, \\
\omega_{2} & =d_{3} d_{4} \cdots d_{r}, \\
& \cdots, \\
\omega_{r-1} & =d_{r}, \\
\omega_{r} & =1 .
\end{aligned}
$$

We replace $\sigma_{1}$ and $\sigma_{2}$ by their conjugates with $\left(\omega_{1}, \omega_{2}, \ldots, \omega_{r}\right)$ and obtain $\sigma_{1}=$ $c$. So $\sigma_{2}=\left(1,1, \ldots, 1, \tau_{1}, 1, \ldots, 1, \tau_{2}, 1, \ldots, 1\right)$, where $\tau_{1}, \tau_{2}$ are involutions in $\operatorname{AGL}_{1}(p)$. They are distinct, for otherwise $\sigma_{1}$ and $\sigma_{2}$ would generate a group containing no subgroup which is transitive on $\Omega_{1}$. Now suppose $r \geq 3$ for a moment. Then there is a power $c^{e}$ of $c$ such that $\sigma_{2}$ and $\sigma_{2}^{c^{e}}$ have only one position in common, where both components are nontrivial. Thus the commutator $\left[\sigma_{2}, \sigma_{2}^{c^{e}}\right]$ has order $p$ on the corresponding $\Omega_{i}$, and is trivial on all the other $\Omega_{j}$ 's. So in this case, again $C_{p}^{r} \leq \dot{N}$. Now if $r=2$ and $C_{p}^{2} \not \leq \dot{N}$, then $p^{2}$ does not divide the order of $\dot{N}$. By Proposition 2.5 we know that $|N / \dot{N}|$ divides $\varphi(2 p)$. If $p=2$, then $G$ has degree 4 , and there is nothing to do. If $p \neq 2$, then $\varphi(2 p)=p-1$, so $p^{2}$ does not divide $|N|$. Thus $U$ and $V$ are Hall subgroups of the solvable group $M=N$, and hence they are conjugate by P. Hall's theorem [14, 6.4.1], a contradiction.

Lemma 3.28. The case $p \neq 4$ does not occur.

Proof. Let $C=C_{p}^{r}$ be the subgroup of $N$ from the previous lemma. As $N \leq$ $\operatorname{AGL}_{1}(p)^{r}$, we conclude that $C$ is the normal Sylow $p$-subgroup of $N$. Thus $M=$ $U C=V C$. Regard $C$ as the $\mathbb{F}_{p}$-vector space $\mathbb{F}_{p}^{r}$. We get that $U \cap C$ and $V \cap C$ are subspaces of codimension 1 . Then, by Kronecker conjugacy, each element in $V \cap C$ has a 0 in at least one component. Suppose $p>2$ for a moment. This forces that there is an $i$ such that all the elements in $V \cap C$ have a 0 in the $i$-th position. Suppose that there is an element $v \in V \cap N$ which has no fixed point on $\Omega_{i}$. Then $v^{p-1}$ lies in $C$. But then there is $c \in V \cap C$, so that $c v$ has no fixed point at all, contrary to Kronecker conjugacy. Thus each element in $V \cap N$ has a fixed point on $\Omega_{i}$. As this group induces a subgroup of $\operatorname{AGL}_{1}(p)$, it then even fixes a point on $\Omega_{i}$. There are three subcases which require different arguments. If $i=1$, then every element of $V$ fixes a point in $\Omega_{1}$ (as by Kronecker conjugacy each element in $V \backslash N$ must fix a point in $\Omega_{1}$ ), so $V$ is not transitive on $\Omega_{1}$, contrary to Lemma 3.4. Now suppose $i>1$. If $M=N$, then $V=V \cap N$, and we have just seen that then $V$ is a point stabilizer, thus conjugate to $U$. Now assume that $M>N$, and choose an $m \in M$ which moves $\Omega_{i}$. Further, as $N_{V}$ fixes a point on $\Omega_{i}$, but is also normal in $M$, it is trivial on $\Omega_{i}$ as well as on $\Omega_{i}^{m}$. Therefore $p^{2}$ divides $\left[C: N_{V} \cap C\right]$. On the other hand, this index equals the size of $C N_{V} / N_{V}$, and the latter group is a subgroup of $M / N_{V}$ with order dividing $p(p-1)$.

So we must have $p=2$. Note that $\dot{G}=C_{r}$, so certainly $C=\dot{N}=N$. In particular, $\dot{U}, \dot{V} \leq N$. We finish as above if $V \cap C$ stabilizes a point. Suppose that this is not the case. Also $\dot{U} \cap C$ and $\dot{V} \cap C$ have index $p$ in $C$; therefore $\dot{U} \cap C=U \cap C$ and $\dot{V} \cap C=V \cap C$. So $\dot{U}$ and $\dot{V}$ are Kronecker conjugate in $\dot{G}$, but not conjugate. In terms of polynomials, we may assume after some linear changes over the complex numbers that $f(X)=\left(X^{2}+1\right)^{r}$ and $g(X)=\left(X^{2}+\gamma\right)^{r}$ for some $\gamma \neq 0$. But $f$ and $g$ have the same branch points by Lemma 2.14. The branch 
points of $f$ are 0 and 1 , and those of $g$ are 0 and $\gamma^{r}$. Thus $\gamma^{r}=1$. Let $\zeta$ satisfy $\zeta^{2}=\gamma$. Then $g(\zeta X)=f(X)$, so $f$ and $g$ are linearly related; therefore $\dot{U}$ and $\dot{V}$ are conjugate by Lemma 2.10 .

Now we are going to rule out the case $M / N_{U}=\mathrm{S}_{4}$. The procedure is similar to the above, but even more complicated. The reader might wonder if there are no better arguments. Maybe there are, but on the other hand we are very close to the actual existence of strongly Kronecker conjugate polynomials. Indeed, if we replace $\mathrm{S}_{4}$ by $D_{4}$, then a careful analysis of the type of difficulties encountered in the following led to an infinite series; see [23].

From now on we assume $M / N_{U}=\mathrm{S}_{4}$, and thus $p=4$.

Lemma 3.29. If $r>3$, then $\mathrm{A}_{4}^{r} \leq N$.

Proof. Again let $\sigma_{1}, \sigma_{2}, \ldots, \sigma_{R}$ be a genus 0 system for $\dot{G}$ with $\overline{\sigma_{1}}$ being an $r$ cycle, and $\sigma_{i} \in N$ for $i \geq 2$. Also, imbed $G$ into $\operatorname{AGL}_{1}(r) \ltimes \mathrm{S}_{4}^{r}$, and write $\sigma_{1}=$ $c\left(d_{1}, d_{2}, \ldots, d_{r}\right)$ with $d_{i} \in \mathrm{S}_{4}$ and $c$ moving $\Omega_{i}$ to $\Omega_{i+1}$. Write $\sigma_{1}^{r}=\left(\delta_{1}, \delta_{2}, \ldots, \delta_{r}\right)$ with $\delta=d_{1} d_{2} \cdots d_{r}$. Note that $\delta_{i}$ is conjugate to $\delta_{1}$ for all $i$. Let $k$ be the number of orbits of $\delta_{1}$ on $\Omega_{1}$. Then (see the procedure above)

$$
\sum_{i=2}^{R} \text { ind } \sigma_{i}=k-1 \leq 3 .
$$

We use the fact from Lemma 3.6 that $N$ induces the full $\mathrm{S}_{4}$ on each $\Omega_{i}$. So to start with the easiest case, suppose that there is a $\sigma_{w}$ which is trivial on all blocks but $\Omega_{i}$, and induces either a transposition, a 3-cycle, or a 4-cycle on $\Omega_{i}$. Then the group generated by the commutators $\left[n, \sigma_{w}\right]$ with $n \in N$ induces $\mathrm{A}_{4}$ on $\Omega_{i}$, and acts trivially on all the other blocks. Conjugating by powers of $\sigma_{1}$ shows that $\mathrm{A}_{4}^{r} \leq N$. Now assume that we cannot find such elements. Then ind $\sigma_{i} \geq 2$ for $i \geq 2$; thus $R=2$. We start with the case $k=4$. We may assume (see the proof of 3.27) that $\sigma_{1}=c$. Write $\sigma_{2}=\left(e_{1}, e_{2}, \ldots, e_{r}\right)$. We go through the different possibilities for $\sigma_{2}$. First assume that $\sigma_{2}$ is trivial on all but three $\Omega_{i}$ 's. Then three of the $e_{i}$ 's are transpositions generating $\mathrm{S}_{4}$, and the other entries are 1. Further, the support of any two of these transpositions has size 2, so their commutator is a 3-cycle. Now, as $r>3$ is a prime, an easy argument shows that there is a power $c^{e}$ of $c$ such that $\sigma_{2}$ and $\sigma_{2}^{c^{e}}$ have only one position with nontrivial entries in common. So the commutator $\left[\sigma_{2}, \sigma_{2}^{c^{e}}\right]$ is a 3 -cycle on some $\Omega_{i}$, and trivial on all the other blocks. As above, we get the assertion. Now suppose that $\sigma_{2}$ is trivial on only two $\Omega_{i}$ 's. As $\sigma_{1} \sigma_{2}$ is a $4 r$-cycle, we get that $\left(\sigma_{1} \sigma_{2}\right)^{r}$ is a 4 -cycle on each $\Omega_{i}$. Thus the product of the two nontrivial components of $\sigma_{2}$ is a 4-cycle. Further, by $(\star)$ we get that these two elements are a transposition and a 3 -cycle with exactly one point moved by both of them. Again, the commutator $\left[\sigma_{2}, \sigma_{2}^{c^{e}}\right]$ is a 3 -cycle on some $\Omega_{i}$ and trivial on the others for a suitable $e$. The case that $\sigma_{2}$ is nontrivial on only one $\Omega_{i}$ cannot occur, as then ind $\sigma_{2}=3$ implies that $\sigma_{2}$ is a 4 -cycle on this $\Omega$, a case we have already dealt with.

So $k \leq 3$. The only two cases not covered yet and allowed by $(\star)$ are $k=3$ and $\sigma_{2}$ is either a double-transposition on one $\Omega_{i}$, or a product of two transpositions on two different $\Omega_{i}$ 's. We first look at the latter case. Let $i_{1}$ and $i_{2}$ be the positions where $\sigma_{2}$ has a transposition. Let $W$ be the group generated by the commutators $\left[\sigma_{2}, n\right], n \in N$. Then $W$ acts as $\mathrm{A}_{4}$ on $\Omega_{i_{1}}$ and $\Omega_{i_{2}}$, and is trivial on the other blocks. Now set $\tau=\sigma_{2}^{\sigma_{2}^{i_{2}-i_{1}}}$. Then $\tau$ has a transposition at $i_{2}$, and (as $r$ is odd) 
is trivial at $i_{1}$. Thus the group generated by $[\tau, n], n \in N$, acts as $\mathrm{A}_{4}$ on $\Omega_{i_{2}}$, and trivially on all the other blocks. Again, $\mathrm{A}_{4}^{r} \leq N$.

So the remaining case is that $\sigma_{2}$ is a double transposition on one block. Geometrically, that means that the polynomial $b$ has a genus 0 system containing a double transposition. But that does not happen - see Lemma 2.9.

Lemma 3.30. The case $M / N_{U}=\mathrm{S}_{4}$ does not occur.

Proof. If $r=2$ or 3 , then this is easily checked using GAP. (One could also refine the above investigation and do this by hand.) Let us assume $r \geq 5$, so $A:=\mathrm{A}_{4}^{r} \leq N$. Of course $A$ is normal in $G$. Therefore $A \not \leq V$. Thus $M=V A$, hence $[A: V \cap A]=4$. Thus $V \cap A$ contains a Sylow 3 -subgroup $S$ of $A$. Then $S \cong C_{3}^{r}$. We first show that for some $i \in\{1,2, \ldots, r\}$ each element of $V \cap N$ has a fixed point on $\Omega_{i}$. Suppose that this is not the case for $i$. Then there is $v \in V \cap N$ which induces either a double transposition or a 4-cycle on $\Omega_{i}$. Let $S_{i}$ be the subgroup of $S \leq V \cap N$, inducing a group of order 3 on $\Omega_{i}$, and acting trivially on all the other blocks. Then $\left[S_{i},\langle v\rangle\right]$ contains the Klein 4-group $K_{4}$ in its regular action. So we end up with $K_{4}^{r} \leq V \cap N$, so there were fixed point free elements in $V \cap N$, contrary to Kronecker conjugacy. So, for some $i$, each element in $V \cap N$ has a fixed point on $\Omega_{i}$. As $V \cap N$ contains $S_{i}$, this group even stabilizes a point in $\Omega_{i}$. Now we finish similarly as in the proof of 3.28. If $i=1$, then, as every element in $V \backslash N$ has a fixed point on $\Omega_{1}$, we get that $V$ is intransitive on $\Omega_{1}$, contrary to Lemma 3.4. If $M=N$, then $V=V \cap N$ fixes a point, so $V$ and $U$ are conjugate. Finally, suppose $M>N$. Then there is $m \in M$ with $\Omega_{i}^{m} \neq \Omega_{i}$. As $N_{V} \unlhd M$ has a fixed point on $\Omega_{i}$, we conclude that $N_{V}$ is trivial on $\Omega_{i}$ as well as on $\Omega_{i}^{m}$. Thus $12^{2}$ divides $\left[A: N_{V} \cap A\right]$. On the other hand, $A N_{V} / N_{V}$ is a subgroup of $M / N_{V}=\mathrm{S}_{4}$, so $\left[A: N_{V} \cap A\right]=\left|A N_{V} / N_{V}\right|$ divides 12 , a contradiction.

3.4. End of the proof. We summarize the result about the groups which have survived. Using Proposition 2.16, we get

Theorem 3.31. Let $K$ be a field of characteristic 0 , and $f, g \in K[X]$ be properly Kronecker conjugate over $K$. Suppose that $f(X)=a(b(X))$ with $a, b \in K[X]$, and that neither a nor $b$ is strongly Kronecker conjugate over $K$ to another polynomial. Then $G$ is one of the following groups: $\mathrm{GL}_{2}(3), \mathrm{GL}_{2}(5) / C_{2}, \mathrm{GL}_{2}(7) / C_{3}$, $\Gamma \mathrm{L}_{2}(9) / C_{4}, \mathrm{GL}_{2}(4), \Gamma \mathrm{L}_{2}(4), \mathrm{GL}_{2}(7) / C_{2}$. The subgroups $U$ and $V$ are (up to conjugation and interchanging) as in Lemma 2.15.

This gives us the part about the degrees in Theorem 1.4. We show that $f$ and $g$ are indeed strongly Kronecker conjugate. An argument similar to the one in the proof of Lemma 3.21 shows that $G=\dot{G}$ except for the case $G=\Gamma L_{2}(4)$. But in this case, $\mathrm{GL}_{2}(4) \leq \dot{G}$. So $\dot{U}$ and $\dot{V}$ are not conjugate in $\dot{G}$ in all cases. The existence part of Theorem 1.4 is the subject of the next section.

So it remains to prove Theorem 1.2. Let $G$ be one of the groups from subsection 3.2. Let $m$ be the degree of $\bar{G}$, and $p$ the degree of $M / N_{U}$. We identify $\Omega$ with the elements from $\mathbb{Z} / p m \mathbb{Z}$, and the blocks $\Omega_{i}$ with the residue classes modulo $m$. So the stabilizer of 0 in $D$ (notation from Proposition 2.5) can be identified with the maps $x \mapsto \alpha x$ for units $\alpha$ in $\mathbb{Z} / p m \mathbb{Z}$. Let $d=(x \mapsto \alpha x)$ be in $N$. This translates to $\alpha \equiv 1(\bmod m)$. Then, as $d$ also fixes a point, we have $d=1$ by $3.14(\mathrm{f})$. So even $\alpha \equiv 1(\bmod p)$. Suppose $p>2$. Then either $\alpha=1+m$ or $\alpha=1+2 m$ is prime to $\mathrm{pm}$ and violates the above conclusion, as $(x \mapsto \alpha x) \in D$ by Proposition 
2.5. Thus $p=2$ and $m$ is odd (for otherwise $\alpha=1+m$ would work). But none of the constellations which appear in Theorem 1.4 has $p=2$ and odd $m$.

\section{EXIstence RESUlts}

This section is devoted to the proof of the existence part of Theorem 1.4. We proceed as follows. Let $G$ be one of the groups in Theorem 3.31 which survived section 3. Further, choose the subgroups $U, V$, and $M$ according to section 2.4. Except for the case $G=\Gamma L_{2}(4)$, we construct a genus 0 system in $G$ of a polynomial. Then, as a consequence of Riemann's existence theorem, there are a number field $E$ and a Galois extension $\Pi$ of $E(t)$ (where $t$ is transcendental) with $G=\operatorname{Gal}(\Pi \mid E(t)$ ), and rational fields $E(x), E(y)$ and $E(z)$ between $E(t)$ and $\Pi$, such that $b(x)=z=$ $b^{\prime}(y)$ and $a(z)=t$ for polynomials $a, b, b^{\prime} \in E[X]$. Then $f(X)=a(b(X))$ and $g(X)=a\left(b^{\prime}(X)\right)$ is a pair of strongly Kronecker conjugate polynomials.

We left the computation of the genus 0 systems to a GAP program. The following list gives the result. In all cases, there are only 2 finite branch points. We give the group $G$ along with the elements $\sigma_{1}, \sigma_{2}, \overline{\sigma_{1}}$ and $\overline{\sigma_{2}}$, where $\overline{\sigma_{i}}$ denotes the action on the cosets of $M$ in $G$, thus giving a genus 0 system of the polynomial $a$. We note that as a result of the computation, the cycle types of the $\sigma$ 's are uniquely given in all cases. This, together with the subsequent computation of explicit examples, gives a certain uniqueness result for pairs of Kronecker conjugate polynomials.

$$
\begin{aligned}
& G=\mathrm{GL}_{2}(3), \quad \sigma_{1}=\left(\begin{array}{lll}
2 & 3
\end{array}\right)\left(\begin{array}{ll}
4 & 8
\end{array}\right)\left(\begin{array}{l}
6 \\
6
\end{array}\right), \sigma_{2}=\left(\begin{array}{lll}
1 & 2 & 4
\end{array}\right)\left(\begin{array}{lll}
5 & 6 & 8
\end{array}\right), \\
& \overline{\sigma_{1}}=\left(\begin{array}{lll}
3 & 4
\end{array}\right), \overline{\sigma_{2}}=\left(\begin{array}{lll}
1 & 3 & 2
\end{array}\right) \text {. } \\
& G=\mathrm{GL}_{2}(5) / C_{2}, \quad \sigma_{1}=(23)(47109)(56118), \\
& \sigma_{2}=\left(\begin{array}{lll}
1 & 4
\end{array}\right)(27)(36)(512) \text {, } \\
& \overline{\sigma_{1}}=\left(\begin{array}{llll}
2 & 5 & 4 & 3
\end{array}\right), \overline{\sigma_{2}}=\left(\begin{array}{ll}
1 & 2
\end{array}\right)\left(\begin{array}{ll}
5 & 6
\end{array}\right) . \\
& G=\mathrm{GL}_{2}(7) / C_{3}, \quad \sigma_{1}=\left(\begin{array}{lll}
5 & 9 & 14
\end{array}\right)(61012)(71116)(81315), \\
& \sigma_{2}=(15)(26)(310)(49)(712)(814)(1516) \text {, } \\
& \overline{\sigma_{1}}=(243)(587), \overline{\sigma_{2}}=(12)(35)(46) \text {. } \\
& G=\Gamma L_{2}(9) / C_{4}, \quad \sigma_{1}=\left(\begin{array}{llll}
1 & 6 & 17 & 4
\end{array}\right)(214)(5111519)(781316)(9121020), \\
& \sigma_{2}=(25)(315)(7 \text { 10)(12 18)(14 20)(17 19), }
\end{aligned}
$$

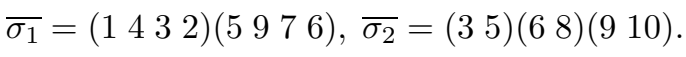

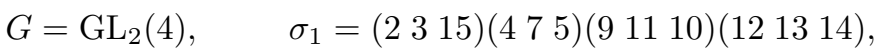

$$
\begin{aligned}
& \sigma_{2}=\left(\begin{array}{ll}
1 & 4)
\end{array}\right)(2 \text { 8)(3 12)(6 9)(7 13)(11 14), } \\
& \overline{\sigma_{1}}=\left(\begin{array}{lll}
2 & 4 & 3
\end{array}\right), \overline{\sigma_{2}}=\left(\begin{array}{ll}
1 & 2
\end{array}\right)\left(\begin{array}{ll}
4 & 5
\end{array}\right) \text {. } \\
& G=\mathrm{GL}_{2}(7) / C_{2}, \quad \sigma_{1}=\left(\begin{array}{lll}
1 & 2 & 4
\end{array}\right)\left(\begin{array}{l}
3 \\
15
\end{array} 12\right)(51814)(72117)(91923) \\
& \text { (11 } 22 \text { 24)(13 } 1620) \text {, } \\
& \sigma_{2}=(15)(27)(34)(622)(816)(921)(1019)(1115)(1318),
\end{aligned}
$$




$$
\overline{\sigma_{1}}=(243)(576), \overline{\sigma_{2}}=\left(\begin{array}{ll}
1 & 2
\end{array}\right)(45)(78) .
$$

We treat these groups case by case. As we do not care about the base field, we are free to make several "without loss of generality" assumptions about the location of branch points and their preimages.

4.0.1. $G=\mathrm{GL}_{2}(3)$. The branching of $a$ is given by $\overline{\sigma_{1}}$ and $\overline{\sigma_{2}}$. Without loss, let 0 be the branch point where the triple point lies above; furthermore, we may assume that this triple point is 0 , and that the other preimage of 0 under $a$ is 1. Thus $a(X)=X^{3}(X-1)$. We find the other finite branch point $b_{1}$ as follows. Compute the discriminant of $a(X)-t$ with respect to $X$; then the zero of that discriminant different from 0 is $b_{1}$. We get $b_{1}=-27 / 256$ as the point corresponding to the transposition. The polynomials $b$ and $b^{\prime}$ have, again without loss, the form $b(X)=X^{2}+\beta$ and $b^{\prime}(X)=X^{2}+\beta^{\prime}$. The branching data given by $\sigma_{1}$ and $\sigma_{2}$ tells us what $\beta$ and $\beta^{\prime}$ look like. Let $\gamma$ and $\gamma^{\prime}$ be the two simple points in the preimage under $a$ of $b_{1}$. The finite branch point of $b(X)$ must be $\gamma$ or $\gamma_{1}$. Now $a(X)-b_{1}=\frac{1}{256}\left(16 X^{2}+8 X+3\right)(4 X-3)^{2}$ tells us that $\gamma$ and $\gamma^{\prime}$ are the zeros of $16 X^{2}+8 X+3$. Thus $b(X)=X^{2}+\frac{-1+\sqrt{-2}}{4}$ and $b^{\prime}(X)=X^{2}+\frac{-1-\sqrt{-2}}{4}$.

4.0.2. $G=\mathrm{GL}_{2}(5) / C_{2}$. To determine $a$, let $b_{1}=0$ correspond to $\overline{\sigma_{1}}$, and let 0 be the quadruple point above 0 . Then $a(X)=X^{4}\left(X^{2}+\eta X+\theta\right)$. As $a$ is indecomposable, $\eta$ must be nonzero; thus $\eta=6$ without loss. There are two solutions for $b_{2}$ different from 0 of the discriminant of $a(X)-t$. To get the correct one, check the factorization of $a(X)-b_{2}$ for these two values. We must have two roots of order 2, and two simple roots. That is only the case if $\theta=25$. Thus $a(X)=X^{4}\left(X^{2}+6 X+25\right)$. (For a different procedure to determine $a$, see [21].) Again set $b(X)=X^{2}+\beta, b^{\prime}(X)=X^{2}+\beta^{\prime}$. From $\sigma_{1}$ and $\sigma_{2}$ we see that $\beta$ and $\beta^{\prime}$ must be one of the two simple points in the fiber $a^{-1}(0)$. So $\beta$ and $\beta^{\prime}$ are the zeros of $X^{2}+6 X+25$. Thus $b(X)=X^{2}-(3-4 i)$ and $b^{\prime}(X)=X^{2}-(3+4 i)$, where $i^{2}=-1$.

4.0.3. $G=\mathrm{GL}_{2}(7) / C_{3}$ and $G=\mathrm{GL}_{2}(7) / C_{2}$. In both cases, we get the same cycle type for the branch cycle description of $a(X)$. The computation is similar as above. We get $a(X)=\left(343 X^{2}-1600+1408 \sqrt{2}\right)^{3}\left(2401 X^{2}-11200 X+9856 \sqrt{2} X+46656-\right.$ $27008 \sqrt{2})$.

The branch point corresponding to $\sigma_{1}$ is 0 . Let $b_{2}$ be the other branch point. We get that $a^{-1}\left(b_{2}\right)$ contains two points of multiplicity 1 . In the case $G=\mathrm{GL}_{2}(7) / C_{3}$ these two points are the finite branch points of $b(X)=X^{2}+\beta$ and $b^{\prime}(X)=$ $X^{2}+\beta^{\prime}$ respectively. Computing yields that $\beta$ and $\beta^{\prime}$ are the different solutions of $16807 X^{2}+58800 X-51744 \sqrt{2} X+150464-84992 \sqrt{2}=0$.

The case $G=\mathrm{GL}_{2}(7) / C_{2}$ is a little different, as now the two points of multiplicity 1 in $a^{-1}(0)$ happen to be the finite branch points $b(X)=X^{3}+\beta$ and $b^{\prime}(X)=$ $X^{3}+\beta^{\prime}$. Thus $\beta$ and $\beta^{\prime}$ are the distinct solutions of $2401 X^{2}-11200 X+9856 \sqrt{2} X+$ $46656-27008 \sqrt{2}=0$.

4.0.4. $G=\Gamma \mathrm{L}_{2}(9) / C_{4}$. The polynomial $a$ has the geometric monodromy group $\mathrm{P} L_{2}(9)$; it has been computed with the given branching data by Matzat $[20,8.7]$. The result is $a(X)=\left(X^{2}-405\right)^{4}\left(X^{2}+50 X+945\right)$. The two points of multiplicity 1 in $a^{-1}(0)$ are the branch points of $b(X)$ and $b^{\prime}(X)$. Thus $b(X)=X^{2}-(25-8 \sqrt{-5})$ and $b^{\prime}(X)=X^{2}-(25+8 \sqrt{-5})$. 
4.0.5. $G=\mathrm{GL}_{2}(4)$. The last case is quite easy. We quickly get the equality $a(X)=$ $X^{3}\left(X^{2}+X+\frac{8}{5}\right)$, and the fact that the branch points of $b(X)$ and $b^{\prime}(X)$ are the points of multiplicity 1 in $a^{-1}(0)$. From this we conclude that $b(X)=X^{2}-\left(\frac{1}{2}-\frac{3}{10} \sqrt{-15}\right)$ and $b^{\prime}(X)=X^{2}-\left(\frac{1}{2}+\frac{3}{10} \sqrt{-15}\right)$.

\section{Conjectures}

We want to state two conjectures. The first one does not look quite hopeless. We have some evidence for it, but are still far away from a solution.

Conjecture 5.1. Let $f, g \in \mathbb{Q}[X]$ be non-constant polynomials which are strongly Kronecker conjugate over $\mathbb{Q}$. Then, up to linear changes over $\mathbb{Q}$, we have $f(X)=$ $h\left(X^{8}\right), g(X)=h\left(16 X^{8}\right)$ for some $h \in \mathbb{Q}[X]$.

All examples of Kronecker conjugate polynomials we have met so far have the even stronger property of arithmetical equivalence. In terms of our groups this means that the permutation characters $\mathbf{1}_{U}^{G}$ and $\mathbf{1}_{V}^{G}$ are the same. This group theoretic property has been investigated by various authors in different contexts; see [16], [18], [25], and the literature given there. Of course, this property can again be translated back to an arithmetical property of the value sets of polynomials on residue fields.

Conjecture 5.2. Let $K$ be a field of characteristic 0 . If $f, g \in K[X]$ are Kronecker conjugate, then they are arithmetically equivalent.

We have examples of finite groups $G$ with Kronecker conjugate subgroups $U$ and $V$, such that $G$ has a cyclic subgroup $Z$ with $G=U Z=V Z$, but $\mathbf{1}_{U}^{G} \neq \mathbf{1}_{V}^{G}$. So if the conjecture should be true, then it is a fancy consequence of the genus 0 condition from subsection 2.2 .

We have no idea how many more types of pairs of Kronecker conjugate polynomials exist. Their determination without further restrictions seems hopeless to us. Still, it would be interesting to exhibit further classes as in Theorem 1.5.

\section{REFERENCES}

1. N. C. Ankeny, C. A. Rogers, A conjecture of Chowla, Ann. of Math. 53 (1951), 541-550; 58 (1953), 591. MR 12:8042; MR 15:210d

2. G. Butler, J. McKay, The transitive groups of degree up to eleven, Comm. Algebra 11(8) (1983), 863-911. MR 84f:20005

3. C. Chevalley, Algebraic Functions of One Variable, Mathematical Surveys VI, AMS, Providence, 1951. MR 13:64a

4. J. Conway, R. Curtis, S. Norton, R. Parker, R. Wilson, Atlas of Finite Groups: Maximal Subgroups and Ordinary Characters for Simple Groups, Clarendon Press, Oxford, New York, 1985. MR 88g:20025

5. W. Feit, On symmetric balance incomplete block designs with doubly transitive automorphism groups, J. Combin. Theory Ser. A, vol. 14 (1973), 221-247. MR 48:5882

6. W. Feit, Some consequences of the classification of finite simple groups, The Santa Cruz conference on finite groups, Proc. Sympos. Pure Math., vol. 37, AMS, Providence, Rhode Island, 1980, 175-181. MR 82c:20019

7. M. Fried, On a conjecture of Schur, Michigan Math. J. 17 (1970), 41-55. MR 41:1688

8. M. Fried, The field of definition of function fields and a problem in the reducibility of polynomials in two variables, Illinois Journal of Mathematics 17 (1973), 128-146. MR 50:329

9. M. Fried, On Hilbert's Irreducibility Theorem, Journal of Number Theory 6 (1974), 211-231. MR 50:2117

10. M. Fried, Rigidity and applications of the classification of simple groups to monodromy, Part II - Applications of connectivity; Davenport and Hilbert-Siegel Problems, preprint. 
11. M. Fried, Extension of constants, rigidity, and the Chowla-Zassenhaus conjecture, Finite Fields and their Applications 1 (1995), 326-359. MR 96i:11120

12. M. Fried, M. Jarden, Field Arithmetic, Springer, Berlin, Heidelberg, 1986. MR 89b:12010

13. M. Fried, R. E. MacRae, On the invariance of chains of fields, Illinois Journal of Mathematics 13 (1969), 165-171. MR 39:179

14. D. Gorenstein, Finite Groups, Harper and Row, New York-Evanston-London, 1968. MR 38:229

15. R. Guralnick, Zeroes of permutation characters with applications to prime splitting and Brauer groups, J. Algebra 131 (1990), 294-302. MR 91j:20038

16. R. Guralnick, Subgroups inducing the same permutation representation, J. Algebra 81 (1983), 312-319. MR 84j:20010

17. W. Jehne, Kronecker classes of algebraic number fields, J. Number Theory 9 (1977), 279-320. MR 56:5499

18. N. Klingen, Zahlkörper mit gleicher Primzerlegung, J. reine angew. Math. 299/300 (1978), 342-384. MR 58:10817

19. L. Kronecker, Über die Irreduzibilität von Gleichungen, Werke II, 85-93; Monatsberichte Deutsche Akademie für Wissenschaft (1880), 155-163.

20. B. H. Matzat, Konstruktion von Zahl-und Funktionenkörpern mit vorgegebener Galoisgruppe, J. Reine Angew. Math. 349 (1984), 179-220. MR 85j:11164

21. P. Müller, Primitive monodromy groups of polynomials. Contemp. Math. 186 (1995), 385-401. MR 96m:20004

22. P. Müller, Reducibility behavior of polynomials with varying coefficients, Israel J. Math. 94 (1996), 59-91. CMP 96:14

23. P. Müller, An infinite series of Kronecker conjugate polynomials, Proc. Amer. Math. Soc. 125 (1997), 1933-1940. CMP 97:10

24. P. Müller, H. Völklein, On a question of Davenport, J. Number Theory 58 (1996), 46-54. MR 97h:12012

25. R. Perlis, On the equation $\zeta_{K}(s)=\zeta_{K^{\prime}}(s)$, J. Number Theory 9 (1977), 342-360. MR 56:5503

26. C. Praeger, Kronecker classes of field extensions of small degree, J. Austr. Math. Soc. (Series A) 50 (1991), 297-315. MR 92m:12004

27. J. F. Ritt, Prime and composite polynomials, Trans. Amer. Math. Soc. 23 (1922), 51-66.

28. G. F. Royle, The transitive groups of degree twelve, J. Symb. Comp.4 (1987), 255-268. MR 89b:20010

29. J. Saxl, On a question of W. Jehne concerning covering subgroups of groups and Kronecker classes of fields, J. London. Math. Soc.(2) 38 (1988), 243-249. MR 90b:11118

30. M. Schönert et. al., GAP - Groups, Algorithms, and Programming, Lehrstuhl D für Mathematik, Rheinisch-Westfälische Techn. Hochschule, Aachen, Germany, fourth edition, 1994.

31. I. Schur, Über den Zusammenhang zwischen einem Problem der Zahlentheorie und einem Satz über algebraische Funktionen, S.-B. Preuss. Akad. Wiss., Phys.-Math. Klasse (1923), 123-134.

32. E. Trost, Zur Theorie der Potenzreste, Nieuw Arch. Wiskunde 18 (1934), 58-61.

33. G. Turnwald, On Schur's conjecture, J. Austr. Math. Soc. (Series A) 58 (1995), 312-357. MR 96a: 11135

34. H. Völklein. Groups as Galois Groups - an Introduction, Cambridge University Press, 1996. CMP 96:17

35. H. Wielandt, Finite Permutation Groups, Academic Press, New York and London, 1964. MR 32:1252

IWR, Universität Heidelberg, D-69120 Heidelberg, Germany

E-mail address: peter.mueller@iwr.uni-heidelberg.de 